\title{
Quality of life measures in Systemic Lupus Erythematosus: A systematic review
}

\author{
M. Radin ${ }^{1,2,3^{*}}$, G. El Hasbani ${ }^{*}$, A. Barinotti ${ }^{1,2,3}$, D. Roccatello ${ }^{1,2,3}$, I. Uthman ${ }^{4}$, \\ A.T. Taher ${ }^{4}$, S. Sciascia ${ }^{1,2,3}$ \\ ${ }^{1}$ Center of Research of Immunopathology and Rare Diseases - Coordination Center of Piemonte and \\ Aosta Valley Network for Rare Diseases, S. Giovanni Bosco Hospital, Department of Clinical and Biological \\ Sciences, University of Turin, Italy; ${ }^{2}$ School of Specialization of Clinical Pathology, Department of Clinical \\ and Biological Sciences, University of Turin, Italy; ${ }^{3}$ Nephrology and Dialysis, Department of Clinical \\ and Biological Sciences, S. Giovanni Bosco Hospital and University of Turin, Italy; ${ }^{4}$ Department of Internal \\ Medicine, American University of Beirut Medical Center, Beirut, Lebanon \\ *These Authors contributed equally to the manuscript
}

\section{SUMMARY}

In this study we systematically investigated the health-related quality of life (HRQoL) tools, which have been most often used over the last five years to evaluate the QoL in patients with systemic lupus erythematosus (SLE), focusing on their items and applications.

A detailed literature search was conducted: the inclusion criteria were as follows:

1) studies including at least 50 patients;

2) studies including at least 25 patients with SLE;

3) quality of life testing with validated measures.

The systematic review was based on 119 studies for a total of 32,449 SLE patients and 3092 controls. A total of 35 different patients-reported quality of life measures, applied in cohorts of patients with SLE, were retrieved with the 36-item Medical Outcome Short Form (SF-36) (63 studies of $119=52.95 \%$ ), Lupus Quality of Life (LupusQoL) (17 studies =14.3\%) and Lupus Patient-Reported Outcome (LupusPRO) (12 studies =10\%) being the most commonly used tools. Overall, this systematic review of the literature indicated that quality of life in patients with SLE appears to be poor and generally lower compared to both the general population and patients with other chronic conditions, as was shown by a few studies that used SF-36 and LupusPRO.

The use of HRQoL scoring in SLE is gaining increasing interest and is used both in randomized controlled trials and in real-life. Future efforts are needed to improve the understanding of the impact of the disease burden on quality of life from the patient's perspective.

Key words: Systemic lupus erythematosus, systematic review, quality of life, measures, scoring, risk factors.

Reumatismo, 2021; 73 (4): 208-228

\section{INTRODUCTION}

ystemic lupus erythematosus (SLE) is an autoimmune chronic disease characterized by multi-organ involvement. In SLE patients, the production of a heterogeneous group of autoantibodies, which bind to their antigens and generate immunecomplexes, can promote inflammatory damage in various organs (1). Additional$1 y$, the resulting wide spectrum of clinical manifestations, as well as the occurrence of several comorbidities (2) and possible drugs side effects can significantly impact on all the aspects of health-related quality of life (HRQoL) in SLE patients, in particular the physical, mental and social dimensions (3). Quality of life in SLE patients is lower compared to both the general population and patients with other chronic diseases, thus leading to a loss of trust in the treating clinicians and poor adherence to therapy $(4,5)$. Hence, it is relevant to consider also HRQoL as an outcome to be evaluated and targeted to improve the management plan and daily life of SLE patients. Consequently, HRQoL is rapidly becoming a fundamental outcome, not only when investigating the well-being of SLE patients, but also when evaluating the efficacy of treatments in randomized controlled trials. 
Over the years, several patients-reported quality of life measures have been developed to assess HRQoL. These measures can be both SLE-specific, such as Lupus Quality of Life Tool (LupusQoL) (6), Quality of Life in Systemic Lupus Erythematosus (L-QoL) (7), Systemic Lupus Erythematosus Quality of Life (SLEQoL) (7), Lupus-Patient-Reported Outcome (LupusPRO) (8) and Lupus Impact Tracker (LIT) (9), or generic, such as the 36-item Medical Outcome Short Form (SF-36) (10), 12-item Medical Outcome Short Form (SF-12) (11) and the Functional Assessment of Chronic Illness Therapy-Fatigue (FACIT-F) (12).

An adequate understanding of their applicability and accuracy in assessing the various dimensions of the HRQoL is crucial, since these tools are based on different measures and have specific advantages and limitations. It is essential to explore the comparability of the different patients-reported quality of life measures to identify the best tool to be used according to the different settings.

Therefore, in this study we investigated systematically the HRQoL tools, which have been most often used over the last five years to evaluate SLE patients, focusing on their items and applications. The aim of our study is to help future researchers investigating QoL in SLE to choose the best tool to use in their studies, learning from past experience gained in worldwide research.

\section{MATERIALS AND METHODS}

A detailed literature search was initially conducted to identify articles that reported findings from clinical studies evaluating the QoL in SLE patients. Key words and subject terms included: ("systemic lupus erythematosus" (MeSH Terms) OR ("systemic lupus erythematosus" (All Fields))) AND ("Quality of life" (MeSH Terms) OR "Quality of life" (All Fields)) AND ("2016/01/04" (PDat): "2021/01/04" (PDat)). The search strategy was applied to Ovid MEDLINE, In-Process and Other Non-Indexed Citation and Ovid Medline for the last 5 years (from January 2016 to January 2021). Abstracts from EULAR and ACR/ARHP Annual Meetings (20152020) were screened and included in the analysis when meeting the inclusion criteria and not replicating studies published elsewhere. Inclusion criteria were:

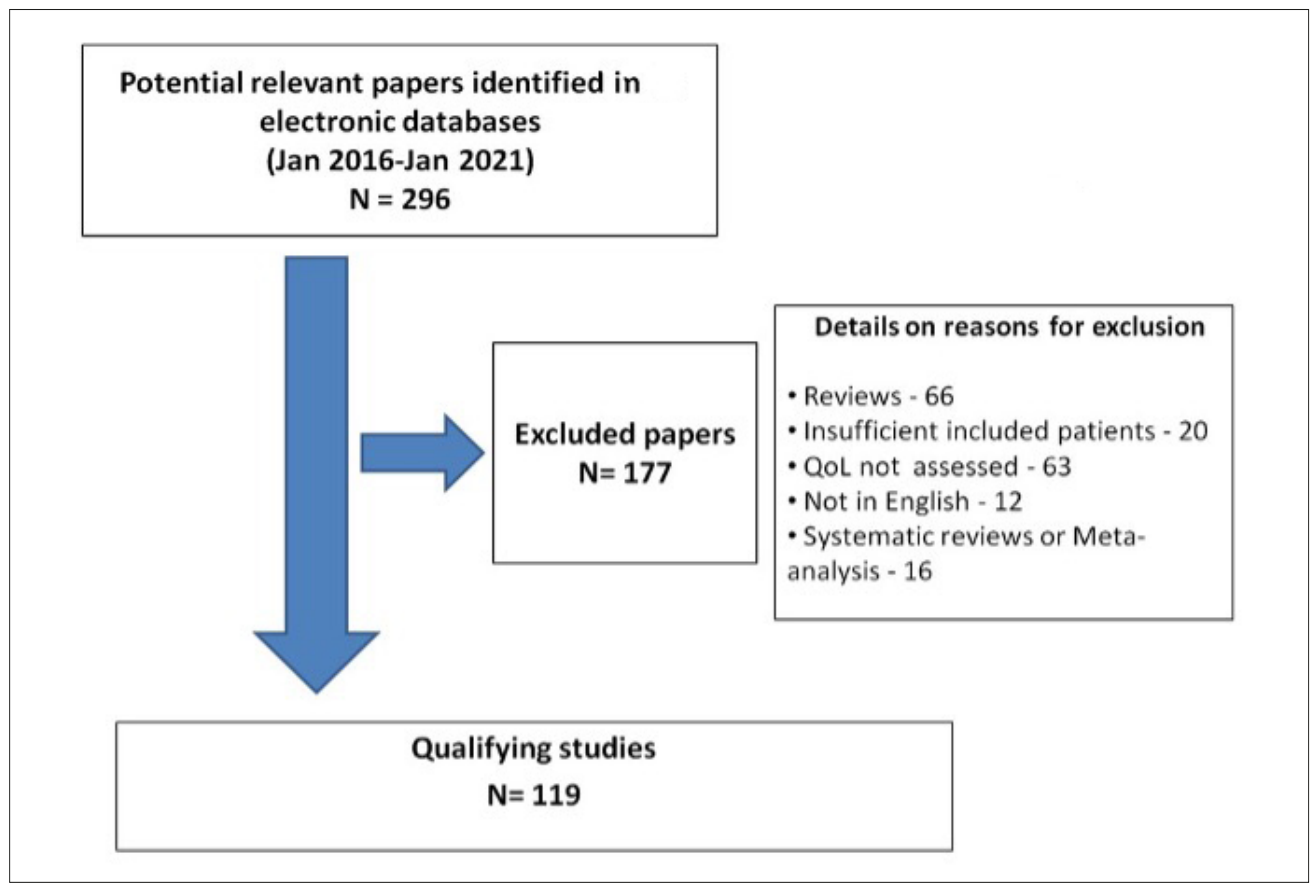

Figure 1 - Literature search strategy and studies' selection. 
1) studies including at least 50 patients;

2) studies including at least 25 patients with SLE;

3) quality of life testing with validated measures [Cross-cultural validated measures were considered, however, for the sake of the study, results are presented with the original scoring measures. Further, different versions of the same score were also considered, but the original version of the score was included in the systematic review].

The studies which met the inclusion criteria were systematically analyzed by two independent reviewers (MR and GEH). Disagreements were resolved by consensus; if it could not be achieved, a third party (SS) provided an assessment of eligibility. Non-English language publications were excluded from the systematic review. The literature search strategy is shown in Figure 1 . This study was performed according to PRISMA guidelines (13-15).

\section{RESULTS}

\section{Literature search}

The systematic review included 119 studies considering a total of 32449 SLE patients and 3092 controls (13-131). The included studies had different designs; in particular: 62 studies out of 119 (52\%) were crosssectional, $24(20.2 \%)$ were case-control, $16(13.5 \%)$ were prospective, $11(9.25 \%)$ were randomized clinical trials, $5(4.2 \%)$ were longitudinal and $1(0.85 \%)$ included a retrospective cohort. Only 11 studies out of $119(9.3 \%)$ enrolled controls who were in total 1779 healthy donors $(57.6 \%)$ and 1313 patients affected by other chronic diseases $(42.5 \%)$, i.e. 605 patients with rheumatoid arthritis, 226 with cutaneous lupus erythematosus, 147 with Sjögren syndrome, 136 with amyopathic dermatomyositis, 120 with systemic sclerosis and 79 with autoimmune blistering diseases. Table I (19-23, 30, 32-144) summarizes the studies included and their main characteristics.

\section{Patients-reported quality of life measures in systemic lupus erythematosus}

A total of 35 different patients-reported quality of life measures applied in cohorts of patients with SLE were retrieved. The 36-item Medical Outcome Short Form (SF-36) (63 studies of $119=52.95 \%)$, Lupus Quality of Life (LupusQoL) (17 studies $=14.3 \%$ ) and Lupus Patient-Reported Outcome (LupusPRO) (12 studies $=10 \%$ )

Table I - Report of the studies included in the systematic review.

\begin{tabular}{|l|l|c|c|c|c|l|l|}
\hline Year & First Author & $\begin{array}{c}\text { Study } \\
\text { design }\end{array}$ & $\begin{array}{c}\text { N. of SLE } \\
\text { patients }\end{array}$ & $\begin{array}{c}\text { N. of } \\
\text { controls }\end{array}$ & $\begin{array}{c}\text { Definition of controls } \\
\text { (i.e. healthy donors) }\end{array}$ & QoL instruments used in the study & Ref. \\
\hline 2014 & Mirbagher et al. & CC & 77 & 840 & Females (20-50 y.o.) & Lupus Quality of Life (LupusQoL) \\
\hline 2015 & Mazzoni et al. & CS & 344 & N/A & N/A & ProblematicSupport Scale (PSS) \\
\hline 2015 & Oner et al. & CS & 113 & N/A & N/A & $\begin{array}{l}\text { Lupus quality of life (LupusQol) and 36-item } \\
\text { ShortForm Health Survey (SF-36) }\end{array}$ \\
\hline 2015 & Wallace et al. & RCT & 203 & N/A & N/A & 36-item ShortForm Health Survey (SF-36) & (36) \\
\hline 2015 & Mazzoni et al. & CS & 344 & N/A & N/A & $\begin{array}{l}\text { Lupus Patient-Reported Outcomes } \\
\text { (LupusPRO) }\end{array}$ \\
\hline 2016 & Hanly et al. & PR & 1827 & N/A & N/A & 36-item ShortForm Health Survey (SF-36) & (38) \\
\hline 2016 & Bostrom et al. & RCT & 35 & N/A & N/A & 36-item ShortForm Health Survey (SF-36) & (39) \\
\hline 2016 & McElhone et al. & PR & 101 & N/A & Nupus quality of life (LupusQol) and 36-item & (22) \\
\hline 2016 & Fidler et al. & CS & 110 & N/A & N/A & 36-item ShortForm Health Survey (SF-36) & (40) \\
\hline 2016 & Mahieu et al. & CS & 123 & N/A & N/A & $\begin{array}{l}\text { Patient Reported Outcomes Measurement } \\
\text { Information System (PROMIS) }\end{array}$ & (41) \\
\hline 2016 & Jolly et al. & RCT & 116 & N/A & N/A & 36-item ShortForm Health Survey (SF-36) & (42) \\
\hline
\end{tabular}




\begin{tabular}{|c|c|c|c|c|c|c|c|}
\hline Year & First Author & $\begin{array}{l}\text { Study } \\
\text { design }\end{array}$ & $\begin{array}{l}\text { N. of SLE } \\
\text { patients }\end{array}$ & $\begin{array}{l}\text { N. of } \\
\text { controls }\end{array}$ & $\begin{array}{l}\text { Definition of controls } \\
\text { (i.e. healthy donors) }\end{array}$ & QoL instruments used in the study & Ref. \\
\hline 2016 & 277 Wang et al. & PR & 60 & N/A & N/A & 36-item ShortForm Health Survey (SF36) & (43) \\
\hline 2016 & Macedo et al. & CS & 216 & $N / A$ & $\mathrm{~N} / \mathrm{A}$ & 36-item ShortForm Health Survey (SF-36) & (44) \\
\hline 2016 & Otter et al. & CS & 131 & N/A & $\mathrm{N} / \mathrm{A}$ & Lupus Quality of Life (LupusQol) & $(45)$ \\
\hline 2016 & Santos et al. & RCT & 120 & $\mathrm{~N} / \mathrm{A}$ & $\mathrm{N} / \mathrm{A}$ & 36-item ShortForm Health Survey (SF-36) & $(46)$ \\
\hline 2016 & 272 Mok et al. & CS & 367 & $\mathrm{~N} / \mathrm{A}$ & $\mathrm{N} / \mathrm{A}$ & 36-item ShortForm Health Survey (SF36) & $(47)$ \\
\hline 2016 & Legge et al. & LG & 273 & $\mathrm{~N} / \mathrm{A}$ & N/A & 36-item ShortForm Health Survey (SF-36) & (48) \\
\hline 2016 & Ramondt et al. & CS & 106 & $\mathrm{~N} / \mathrm{A}$ & N/A & $\begin{array}{l}\text { Brief Illness Perception Questionnaire } \\
\text { (Brief IPQ) }\end{array}$ & (49) \\
\hline 2016 & Mazzoni et al. & LG & 162 & $\mathrm{~N} / \mathrm{A}$ & N/A & $\begin{array}{l}\text { Lupus Patient-Reported Outcomes } \\
\text { (LupusPRO) }\end{array}$ & (50) \\
\hline 2016 & Sebastiani et al. & PR & 122 & $\mathrm{~N} / \mathrm{A}$ & N/A & Visual analogue scale (VAS) & (51) \\
\hline 2016 & Piga et al. & PR & 80 & $\mathrm{~N} / \mathrm{A}$ & N/A & 36-item ShortForm Health Survey (SF-36) & (52) \\
\hline 2016 & Williams et al. & LG & 50 & $\mathrm{~N} / \mathrm{A}$ & N/A & $\begin{array}{l}\text { 36-item ShortForm Health Survey (SF-36) } \\
\text { and Functional Assessment of Chronic } \\
\text { Illness Therapy-Fatigue (FACIT-F) }\end{array}$ & (53) \\
\hline 2016 & Devilliers et al. & LG & 325 & $\mathrm{~N} / \mathrm{A}$ & $\mathrm{N} / \mathrm{A}$ & $\begin{array}{l}\text { Lupus Impact Tracker (LIT), 36-item } \\
\text { ShortForm Health Survey (SF-36), } \\
\text { and Lupus Quality of Life (LupusQL) }\end{array}$ & (21) \\
\hline 2016 & $\begin{array}{l}\text { Lopez-Pina } \\
\text { et al. }\end{array}$ & CS & 232 & $\mathrm{~N} / \mathrm{A}$ & N/A & EuroQol-5 dimension (EQ-5D) & (54) \\
\hline 2016 & Pinto et al. & CS & 39 & $\mathrm{~N} / \mathrm{A}$ & $\mathrm{N} / \mathrm{A}$ & 36-item ShortForm Health Survey (SF-36) & (55) \\
\hline 2016 & Escobar et al. & CS & 147 & $\mathrm{~N} / \mathrm{A}$ & $\mathrm{N} / \mathrm{A}$ & $\begin{array}{l}\text { Lupus quality of life (LupusQol) } \\
\text { and EuroQol (EQ-5D) }\end{array}$ & (56) \\
\hline 2016 & Chen et al. & CS & 541 & $\mathrm{~N} / \mathrm{A}$ & $\mathrm{N} / \mathrm{A}$ & $\begin{array}{l}\text { 36-item ShortForm Health Survey (SF-36) } \\
\text { and EuroQol-5 dimension (EQ-5D) }\end{array}$ & (57) \\
\hline 2016 & Inoue et al. & CS & 205 & $\mathrm{~N} / \mathrm{A}$ & $\mathrm{N} / \mathrm{A}$ & $\begin{array}{l}\text { Lupus Patient-Reported Outcomes } \\
\text { (LupusPRO) }\end{array}$ & (58) \\
\hline 2017 & Jones et al. & $L G$ & 100 & $\mathrm{~N} / \mathrm{A}$ & $\mathrm{N} / \mathrm{A}$ & $\begin{array}{l}\text { Pediatric Quality of Life Generic Core } \\
\text { Scale (PedsQL-GC), Pediatric Quality } \\
\text { of Life Rheumatology Module (PedsQL-RM), } \\
\text { Functional disability inventory (FDI), } \\
\text { Simple Measure of Impact of Lupus } \\
\text { Erythematosus in Youngsters (SMILEY), } \\
\text { Childhood Health Assessment } \\
\text { Questionnaire (C-HAQ), and Child } \\
\text { Health Questionnaire (CHQ-PF50) }\end{array}$ & (59) \\
\hline 2017 & Moorthy et al. & PR & 467 & N/A & $\mathrm{N} / \mathrm{A}$ & $\begin{array}{l}\text { Simple Measure of Impact of Lupus in } \\
\text { Youngsters (SMILEY) and Pediatric Quality } \\
\text { of Life (PedsQL) }\end{array}$ & (32) \\
\hline 2017 & Antony et al. & CS & 73 & $\mathrm{~N} / \mathrm{A}$ & $\mathrm{N} / \mathrm{A}$ & Lupus Impact Tracker (LIT) & (60) \\
\hline 2017 & Brandt et al. & CS & 860 & $\mathrm{~N} / \mathrm{A}$ & $\mathrm{N} / \mathrm{A}$ & $\begin{array}{l}\text { 12-item ShortForm health survey } \\
\text { questionnaire (SF-12) and Lupus Impact } \\
\text { Tracker (LIT) }\end{array}$ & (61) \\
\hline 2017 & $\begin{array}{l}\text { Yilmaz-Oner } \\
\text { et al. }\end{array}$ & $\mathrm{CC}$ & 99 & 71 & No chronic diseases & $\begin{array}{l}\text { 36-item ShortForm Health Survey (SF36) } \\
\text { and Multidimensional Assessment of } \\
\text { Fatigue (MAF) }\end{array}$ & (62) \\
\hline 2017 & Calderon et al. & CS & 101 & $\mathrm{~N} / \mathrm{A}$ & N/A & $\begin{array}{l}\text { 12-item ShortForm health survey } \\
\text { questionnaire (SF-12) }\end{array}$ & (63) \\
\hline
\end{tabular}




\begin{tabular}{|c|c|c|c|c|c|c|c|}
\hline Year & First Author & $\begin{array}{l}\text { Study } \\
\text { design }\end{array}$ & $\begin{array}{l}\text { N. of SLE } \\
\text { patients }\end{array}$ & $\begin{array}{l}\text { N. of } \\
\text { controls }\end{array}$ & $\begin{array}{l}\text { Definition of controls } \\
\text { (i.e. healthy donors) }\end{array}$ & QoL instruments used in the study & Ref. \\
\hline 2017 & Lai et al. & CS & 333 & N/A & $\mathrm{N} / \mathrm{A}$ & $\begin{array}{l}\text { 29-item short form profile (PROMIS-29) and } \\
\text { Quality of Life in Neurological Disorders } \\
\text { (Neuro-QoL) }\end{array}$ & (64) \\
\hline 2017 & Azizoddin et al. & CS & 136 & N/A & N/A & $\begin{array}{l}\text { Lupus Patient-Reported Outcomes } \\
\text { (LupusPRO) }\end{array}$ & (65) \\
\hline 2017 & $\begin{array}{l}\text { Etchegaray- } \\
\text { Morales et al. }\end{array}$ & CS & 138 & $\mathrm{~N} / \mathrm{A}$ & $\mathrm{N} / \mathrm{A}$ & Lupus Quality of Life (LupusQol) & (66) \\
\hline 2017 & Inoue et al. & CS & 205 & $\mathrm{~N} / \mathrm{A}$ & $\mathrm{N} / \mathrm{A}$ & $\begin{array}{l}\text { Pittsburgh Sleep Quality Index (PSQI), } \\
\text { 12-item ShortForm Health Survey (SF-12), } \\
\text { and Lupus Patient-Reported Outcomes } \\
\text { (LupusPRO) }\end{array}$ & (67) \\
\hline 2017 & Schneider et al. & PR & 569 & $\mathrm{~N} / \mathrm{A}$ & $\mathrm{N} / \mathrm{A}$ & Lupus Impact Tracker (LIT) & (68) \\
\hline 2017 & $\begin{array}{l}\text { Stummvoll } \\
\text { et al. }\end{array}$ & CS & 118 & $\mathrm{~N} / \mathrm{A}$ & $\mathrm{N} / \mathrm{A}$ & Self-developed questionnaire & (69) \\
\hline 2017 & Chaigne et al. & $\mathrm{CC}$ & 267 & 267 & $\begin{array}{l}\text { Diagnosed with } \\
\text { rheumatoid arthritis as } \\
\text { per the ACR criteria }\end{array}$ & 36-item ShortForm Health Survey (SF-36) & (70) \\
\hline 2017 & 212 Taniet al. & CS & 50 & $\mathrm{~N} / \mathrm{A}$ & $\mathrm{N} / \mathrm{A}$ & $\begin{array}{l}\text { Visual Analog Scale (VAS), Patient's } \\
\text { perception of global disease activity and } \\
\text { general health (GH), and Health Assessment } \\
\text { Questionnaire (HAQ) }\end{array}$ & (71) \\
\hline 2017 & Nantes et al. & CS & 78 & $\mathrm{~N} / \mathrm{A}$ & $\mathrm{N} / \mathrm{A}$ & 36-item ShortForm Health Survey (SF-36) & (19) \\
\hline 2017 & Golder et al. & CS & 1422 & $\mathrm{~N} / \mathrm{A}$ & N/A & $\begin{array}{l}\text { 36-item ShortForm Health Survey version } 2 \\
\text { (SF-36v2) }\end{array}$ & (72) \\
\hline 2017 & Chaigne et al. & CS & 252 & $\mathrm{~N} / \mathrm{A}$ & $\mathrm{N} / \mathrm{A}$ & 36-item ShortForm Health Survey (SF-36) & (73) \\
\hline 2017 & Magro et al. & PR & 232 & $\mathrm{~N} / \mathrm{A}$ & $\mathrm{N} / \mathrm{A}$ & 36-item ShortForm Health Survey (SF-36) & (74) \\
\hline 2017 & Kent et al. & CS & 121 & $\mathrm{~N} / \mathrm{A}$ & $\mathrm{N} / \mathrm{A}$ & Lupus Quality of Life (LupusQol) & (75) \\
\hline 2017 & Kasturi et al. & CS & 162 & $\mathrm{~N} / \mathrm{A}$ & $\mathrm{N} / \mathrm{A}$ & $\begin{array}{l}\text { 36-item ShortForm Health Survey } \\
\text { (SF-36), Lupus quality of life (LupusQol), and } \\
\text { Patient Reported Outcomes Measurement } \\
\text { Information System (PROMIS) }\end{array}$ & (76) \\
\hline 2017 & Monahan et al. & PR & 248 & $\mathrm{~N} / \mathrm{A}$ & $\mathrm{N} / \mathrm{A}$ & 36-item ShortForm Health Survey (SF-36) & (77) \\
\hline 2017 & Mok et al. & $\mathrm{RC}$ & 769 & N/A & N/A & $\begin{array}{l}\text { 36-item ShortForm Health Survey (SF-36) } \\
\text { and Lupus Patient-Reported Outcomes } \\
\text { (LupusPRO) }\end{array}$ & (78) \\
\hline 2017 & Uzuner et al. & $\mathrm{CC}$ & 41 & 49 & $\begin{array}{l}\text { Healthy children and } \\
\text { adolescents from local } \\
\text { community }\end{array}$ & $\begin{array}{l}\text { Pediatric quality of life inventory child } \\
\text { versions (PedsQL-C) }\end{array}$ & (79) \\
\hline 2017 & $\begin{array}{l}\text { Meseguer- } \\
\text { Henarejos et al. }\end{array}$ & CS & 223 & N/A & N/A & Lupus quality of life (LupusQoL) & (80) \\
\hline 2017 & Duruöz et al. & CS & 55 & N/A & $\mathrm{N} / \mathrm{A}$ & $\begin{array}{l}\text { SLE Quality of Life Questionnaire (L-QoL), } \\
\text { Nottingham Health Profile (NHP), and Health } \\
\text { Assessment Questionnaire (HAQ) }\end{array}$ & (81) \\
\hline 2017 & Shamekhi et al. & RCT & 68 & N/A & N/A & 12-item ShortForm Health Survey (SF-12) & (82) \\
\hline 2017 & Correa et al. & $\mathrm{CC}$ & 75 & 78 & $\begin{array}{l}\text { Subjects without known } \\
\text { rheumatic diseases, } \\
\text { randomly assigned from } \\
\text { a population of workers } \\
\text { of the public health } \\
\text { services or family and } \\
\text { friends of SLE patients }\end{array}$ & Oral health impact profile (OHIP-49) & (83) \\
\hline
\end{tabular}




\begin{tabular}{|c|c|c|c|c|c|c|c|}
\hline Year & First Author & $\begin{array}{l}\text { Study } \\
\text { design }\end{array}$ & $\begin{array}{l}\text { N. of SLE } \\
\text { patients }\end{array}$ & $\begin{array}{l}\text { N. of } \\
\text { controls }\end{array}$ & $\begin{array}{l}\text { Definition of controls } \\
\text { (i.e. healthy donors) }\end{array}$ & QoL instruments used in the study & Ref. \\
\hline 2017 & Contentti et al. & CS & 191 & N/A & N/A & 36-item ShortForm Health Survey (SF-36) & (84) \\
\hline 2017 & Margiotta et al. & CS & 100 & $\mathrm{~N} / \mathrm{A}$ & $\mathrm{N} / \mathrm{A}$ & 36-item ShortForm Health Survey (SF-36) & (85) \\
\hline 2017 & $\begin{array}{l}\text { Muhammed } \\
\text { et al. }\end{array}$ & CS & 101 & N/A & N/A & EuroQoL-5dimension (EuroQol-5D) & (86) \\
\hline 2017 & Hersh et al. & CS & 939 & N/A & $\mathrm{N} / \mathrm{A}$ & $\begin{array}{l}\text { Childhood Health Assessment } \\
\text { Questionnaire (CHAQ) }\end{array}$ & (87) \\
\hline 2018 & Cosatti et al. & CS & 130 & N/A & N/A & $\begin{array}{l}\text { Lupus quality of life (LupusQol) and Visual } \\
\text { Analog Scale (VAS) }\end{array}$ & (23) \\
\hline 2018 & Piga et al. & $\mathrm{CC}$ & 101 & 72 & $\begin{array}{l}\text { Healthy controls enrolled } \\
\text { from hospital staff ( } n=37) \\
\text { and patients classified } \\
\text { with rheumatoid arthritis } \\
\text { according to the } 1987 \\
\text { ACR criteria }(n=35)\end{array}$ & $\begin{array}{l}\text { Functional Assessment of Chronic Illness } \\
\text { Therapy-Fatigue (FACIT-F), 36-item } \\
\text { ShortForm Health Survey (SF-36), and } \\
\text { Heath Assessment Questionnaire (HAQ) }\end{array}$ & (88) \\
\hline 2018 & ludici et al. & CS & 83 & N/A & N/A & $\begin{array}{l}\text { 36-item ShortForm Health Survey (SF-36), } \\
\text { Health Assessment Questionnaire Disability } \\
\text { Index (HAQ-DI), and Hospital Anxiety and } \\
\text { Depression Scale }\end{array}$ & (89) \\
\hline 2018 & Donnelly et al. & CS & 50 & $\mathrm{~N} / \mathrm{A}$ & $\mathrm{N} / \mathrm{A}$ & $\begin{array}{l}\text { Pediatric Quality Of Life Inventory Generic } \\
\text { Core (PedsQL-GC) and Pediatric Quality of } \\
\text { Life Rheumatology Module (PedsQL-RM) }\end{array}$ & (90) \\
\hline 2018 & Zeng et al. & CC & 104 & 104 & $\begin{array}{l}\text { Healthy relatives } \\
\text { of SLE patients }\end{array}$ & 36-item ShortForm Health Survey (SF-36) & (91) \\
\hline 2018 & Jolly et al. & CS & 1259 & N/A & N/A & $\begin{array}{l}\text { Lupus Patient-Reported Outcome } \\
\text { (LupusPRO) }\end{array}$ & (92) \\
\hline 2018 & Golder et al. & CS & 105 & $\mathrm{~N} / \mathrm{A}$ & $\mathrm{N} / \mathrm{A}$ & Lupus Impact Tracker (LIT) & (93) \\
\hline 2018 & Baba et al. & CS & 233 & $\mathrm{~N} / \mathrm{A}$ & $\mathrm{N} / \mathrm{A}$ & 36-item ShortForm Health Survey (SF-36) & (94) \\
\hline 2018 & Lee et al. & PR & 244 & $\mathrm{~N} / \mathrm{A}$ & $\mathrm{N} / \mathrm{A}$ & 36-item ShortForm Health Survey (SF-36) & (95) \\
\hline 2018 & Azizoddin et al. & CS & 131 & $\mathrm{~N} / \mathrm{A}$ & N/A & $\begin{array}{l}\text { 36-item ShortForm Health Survey (SF-36), } \\
\text { Functional Assessment of Chronic IIIness } \\
\text { Therapy-Fatigue (FACIT-F), Insomnia } \\
\text { Severity Index (ISI), Patient Health } \\
\text { Questionnaire-9 (PHQ-9), and Lupus } \\
\text { Patient-Reported Outcome (LupusPRO) }\end{array}$ & (96) \\
\hline 2018 & Jiang et al. & CS & 223 & N/A & $\mathrm{N} / \mathrm{A}$ & $\begin{array}{l}\text { Systemic Lupus Erythematosus-Specific } \\
\text { Quality of Life Questionnaire (SLEQOL) and } \\
\text { 36-item ShortForm Health Survey (SF-36) }\end{array}$ & (97) \\
\hline 2018 & $\begin{array}{l}\text { Elera- } \\
\text { Fitzcarrald } \\
\text { et al. }\end{array}$ & PR & 277 & N/A & $\mathrm{N} / \mathrm{A}$ & Lupus quality of Life (Lupus-QoL) & (98) \\
\hline 2018 & Azizoddin et al. & $\mathrm{CC}$ & 116 & N/A & $\mathrm{N} / \mathrm{A}$ & $\begin{array}{l}\text { Functional Assessment of Chronic Illness } \\
\text { Therapy-Fatigue (FACIT-F), and Lupus } \\
\text { Patient-reported Outcome (LupusPRO) }\end{array}$ & (99) \\
\hline 2018 & $\begin{array}{l}\text { Tsang-A-Sjoe } \\
\text { et al. }\end{array}$ & CC & 154 & N/A & N/A & 36-item ShortForm Health Survey (SF-36) & $(100)$ \\
\hline 2018 & $\begin{array}{l}\text { Pereira E Silva } \\
\text { et. al }\end{array}$ & CC & 68 & 27 & Primary Sjögren & $\begin{array}{l}\text { Overactive bladder questionnaire short form } \\
\text { (OABq-SF) }\end{array}$ & (101) \\
\hline 2018 & Strand et al. & RCT & 268 & N/A & N/A & $\begin{array}{l}\text { Functional Assessment of Chronic Illness } \\
\text { Therapy-Fatigue (FACIT-F), 36-item } \\
\text { ShortForm Health Survey (SF-36) }\end{array}$ & (102) \\
\hline 2018 & Wang et al. & $\mathrm{CC}$ & 60 & $\mathrm{~N} / \mathrm{A}$ & $\mathrm{N} / \mathrm{A}$ & 36-item ShortForm Health Survey (SF-36) & (103) \\
\hline
\end{tabular}




\begin{tabular}{|c|c|c|c|c|c|c|c|}
\hline Year & First Author & $\begin{array}{l}\text { Study } \\
\text { design }\end{array}$ & $\begin{array}{l}\text { N. of SLE } \\
\text { patients }\end{array}$ & $\begin{array}{l}\text { N. of } \\
\text { controls }\end{array}$ & $\begin{array}{l}\text { Definition of controls } \\
\text { (i.e. healthy donors) }\end{array}$ & QoL instruments used in the study & Ref. \\
\hline 2018 & Tarazi et al. & CC & 165 & 441 & $\begin{array}{c}\text { Cutaneous lupus } \\
\text { erythematosus } \\
(n=226), \text { Amyopathic } \\
\text { dermatomyositis }(n=136) \\
\text { and autoimmune } \\
\text { blistering diseases }(n=79)\end{array}$ & 36-item ShortForm Health Survey (SF-36) & (104) \\
\hline 2018 & Groot et al. & $\mathrm{CC}$ & 111 & N/A & $\mathrm{N} / \mathrm{A}$ & 36-item ShortForm Health Survey (SF-36) & (105) \\
\hline 2018 & Xie et al. & RCT & 125 & $\mathrm{~N} / \mathrm{A}$ & $\mathrm{N} / \mathrm{A}$ & 36-item ShortForm Health Survey (SF-36) & $(106)$ \\
\hline 2018 & Parodis et al. & PR & 69 & $\mathrm{~N} / \mathrm{A}$ & $\mathrm{N} / \mathrm{A}$ & $\begin{array}{l}\text { 36-item ShortForm Health Survey } \\
\text { (SF-36), Functional Assessment of Chronic } \\
\text { Illness Therapy-Fatigue (FACIT-F), EuroQol } \\
\text { 5-dimension (EQ-5D) }\end{array}$ & (107) \\
\hline 2018 & Jolly et al. & CC & 1803 & $\mathrm{~N} / \mathrm{A}$ & N/A & $\begin{array}{l}\text { Lupus Patient-Reported Outcome } \\
\text { (LupusPRO) }\end{array}$ & (108) \\
\hline 2018 & $\begin{array}{l}\text { Figueiredo- } \\
\text { Braga et al. }\end{array}$ & CC & 72 & N/A & $\mathrm{N} / \mathrm{A}$ & $\begin{array}{l}\text { Chalder Fatigue Scale (CFS), 36-item } \\
\text { ShortForm Health Survey (SF-36), and } \\
\text { Fatigue Severity Scale (FSS) }\end{array}$ & (109) \\
\hline 2018 & Waldheim et al. & PR & 84 & $\mathrm{~N} / \mathrm{A}$ & N/A & $\begin{array}{l}\text { Multidimensional Assessment of Fatigue } \\
\text { (MAF), and 36-item ShortForm Health } \\
\text { Survey (SF-36) }\end{array}$ & (110) \\
\hline 2018 & $\begin{array}{l}\text { Pascual-ramos } \\
\text { et al. }\end{array}$ & $\mathrm{CC}$ & 245 & 183 & Rheumatoid arthritis & 36-item ShortForm Health Survey (SF-36) & (111) \\
\hline 2018 & Merrill et al. & RCT & 442 & $\mathrm{~N} / \mathrm{A}$ & $\mathrm{N} / \mathrm{A}$ & Lupus quality of life (Lupus QoL) & $(112)$ \\
\hline 2018 & Margiotta et al. & $\mathrm{CC}$ & 93 & $\mathrm{~N} / \mathrm{A}$ & N/A & $\begin{array}{l}\text { 36-item ShortForm Health Survey (SF-36), } \\
\text { and Functional Assessment of Chronic } \\
\text { Illness Therapy-Fatigue (FACIT-F) }\end{array}$ & (113) \\
\hline 2018 & Kasturi et al. & CC & 204 & N/A & $\mathrm{N} / \mathrm{A}$ & $\begin{array}{l}\text { Lupus Quality of Life (Lupus QoL), and 36- } \\
\text { item ShortForm Health Survey (SF-36) }\end{array}$ & (114) \\
\hline 2018 & Zhao et al. & $\mathrm{CC}$ & 118 & $\mathrm{~N} / \mathrm{A}$ & $\mathrm{N} / \mathrm{A}$ & Lupus Quality of Life (LupusQoL) & $(115)$ \\
\hline 2018 & $\begin{array}{l}\text { Nowicka-Sauer } \\
\text { et al. }\end{array}$ & CC & 80 & $\mathrm{~N} / \mathrm{A}$ & $\mathrm{N} / \mathrm{A}$ & Fatigue Severity Scale (FSS) & (116) \\
\hline 2018 & Aziz et al. & CC & 91 & N/A & N/A & $\begin{array}{l}\text { 36-item ShortForm Health Survey (SF- } \\
\text { 36), and Systemic Lupus Erythematosus } \\
\text { specific-quality of life instrument (SLEQOL) }\end{array}$ & (117) \\
\hline 2019 & Seguier et al. & CS & 57 & N/A & $\mathrm{N} / \mathrm{A}$ & $\begin{array}{l}\text { 36-item ShortForm Health Survey } \\
\text { (SF-36) }\end{array}$ & (118) \\
\hline 2019 & Margiotta et al. & CS & 136 & N/A & $\mathrm{N} / \mathrm{A}$ & $\begin{array}{l}\text { 36-item ShortForm Health Survey } \\
\text { (SF-36) }\end{array}$ & (119) \\
\hline 2019 & Jolly et al. & RCT & 867 & $\mathrm{~N} / \mathrm{A}$ & N/A & $\begin{array}{l}\text { 36-item ShortForm Health Survey (SF-36), } \\
\text { andFunctional Assessment of Chronic } \\
\text { Illness Therapy-Fatigue (FACIT-F) }\end{array}$ & (120) \\
\hline 2019 & Pinto et al. & CS & 144 & $\mathrm{~N} / \mathrm{A}$ & $\mathrm{N} / \mathrm{A}$ & $\begin{array}{l}\text { Lupus Patient-Reported Outcome } \\
\text { (LupusPRO) }\end{array}$ & (121) \\
\hline 2019 & $\begin{array}{l}\text { Corneloup } \\
\text { et al. }\end{array}$ & PR & 336 & N/A & N/A & $\begin{array}{l}\text { Lupus Quality of Life (LupusQoL), and } \\
\text { Systemic Lupus Erythematosus-specific } \\
\text { quality of life instrument (SLEQOL) }\end{array}$ & (20) \\
\hline 2019 & Pinto et al. & CS & 112 & N/A & N/A & $\begin{array}{l}\text { Patient Health Questionnaire } 9 \text { (PHQ9), } \\
\text { Generalized Anxiety Disorder } 7 \text { (GAD7), and } \\
\text { Fatigue Severity Scale (FSS) }\end{array}$ & (122) \\
\hline 2019 & $\begin{array}{l}\text { Gholizadeh } \\
\text { et al. }\end{array}$ & CS & 135 & N/A & $\mathrm{N} / \mathrm{A}$ & $\begin{array}{l}\text { Lupus Patient-Reported Outcome } \\
\text { (LupusPRO), Hospital anxiety and } \\
\text { depression scale (HADS) }\end{array}$ & (123) \\
\hline
\end{tabular}




\begin{tabular}{|c|c|c|c|c|c|c|c|}
\hline Year & First Author & $\begin{array}{l}\text { Study } \\
\text { design }\end{array}$ & $\begin{array}{l}\text { N. of SLE } \\
\text { patients }\end{array}$ & $\begin{array}{l}\text { N. of } \\
\text { controls }\end{array}$ & $\begin{array}{l}\text { Definition of controls } \\
\text { (i.e. healthy donors) }\end{array}$ & QoL instruments used in the study & Ref. \\
\hline 2019 & Wu et al. & $\mathrm{RCT}$ & 76 & $\mathrm{~N} / \mathrm{A}$ & N/A & $\begin{array}{l}\text { Fatigue Severity Scale (FSS), and 36-item } \\
\text { ShortForm Health Survey (SF36) }\end{array}$ & (124) \\
\hline 2019 & Živković et al. & CS & 83 & $\mathrm{~N} / \mathrm{A}$ & $\mathrm{N} / \mathrm{A}$ & $\begin{array}{l}\text { Fatigue Severity Scale (FSS), and 36-item } \\
\text { ShortForm Health Survey (SF36) }\end{array}$ & (125) \\
\hline 2019 & Kasturi et al. & CS & 223 & $\mathrm{~N} / \mathrm{A}$ & $\mathrm{N} / \mathrm{A}$ & $\begin{array}{l}\text { PROMIS Global } \\
\text { Health Short Form (PROMIS10), and 36- } \\
\text { item ShortForm Health Survey (SF-36) }\end{array}$ & (126) \\
\hline 2019 & $\begin{array}{l}\text { Poomsalood } \\
\text { et al. }\end{array}$ & CS & 237 & $\mathrm{~N} / \mathrm{A}$ & N/A & $\begin{array}{l}\text { Systemic Lupus Erythematosus-specific } \\
\text { Quality of Life questionnaire (SLEQoL) }\end{array}$ & (127) \\
\hline 2019 & Ugarte-Gil et al. & CS & 483 & $\mathrm{~N} / \mathrm{A}$ & $\mathrm{N} / \mathrm{A}$ & $\begin{array}{l}\text { 36-item ShortForm Health Survey } \\
\text { (SF-36) }\end{array}$ & (128) \\
\hline 2019 & Ugarte-Gil et al. & CS & 243 & N/A & N/A & Lupus Quality of Life (LupusQoL) & (129) \\
\hline 2019 & Margiotta et al. & CS & 130 & $\mathrm{~N} / \mathrm{A}$ & $\mathrm{N} / \mathrm{A}$ & $\begin{array}{l}\text { 36-item ShortForm Health Survey (SF-36), } \\
\text { and Functional Assessment of Chronic } \\
\text { Illness Therapy-Fatigue (FACIT-F) }\end{array}$ & (130) \\
\hline 2019 & Goswami et al. & CS & 126 & $\mathrm{~N} / \mathrm{A}$ & $\mathrm{N} / \mathrm{A}$ & $\begin{array}{l}\text { Fatigue Severity Scale (FSS); and 12-item } \\
\text { ShortForm Health Survey (SF-12) }\end{array}$ & (131) \\
\hline 2019 & Geng et al. & CS & 201 & $\mathrm{~N} / \mathrm{A}$ & $\mathrm{N} / \mathrm{A}$ & $\begin{array}{l}\text { Fatigue Severity Scale (FSS) } 9 \\
\text { and } 7 \text { items }\end{array}$ & (132) \\
\hline 2019 & $\begin{array}{l}\text { Abu Bakar } \\
\text { et al. }\end{array}$ & CS & 215 & $\mathrm{~N} / \mathrm{A}$ & N/A & $\begin{array}{l}\text { 36-item ShortForm Health Survey } \\
\text { (SF-36) }\end{array}$ & (133) \\
\hline 2019 & $\begin{array}{l}\text { Horisberger } \\
\text { et al. }\end{array}$ & CS & 73 & $\mathrm{~N} / \mathrm{A}$ & $\mathrm{N} / \mathrm{A}$ & $\begin{array}{l}\text { 36-item ShortForm Health Survey (SF-36), } \\
\text { and Fatigue Assessment Scale (FAS) }\end{array}$ & (134) \\
\hline 2019 & $\begin{array}{l}\text { Romànlvorra } \\
\text { et al. }\end{array}$ & CS & 190 & $\mathrm{~N} / \mathrm{A}$ & $\mathrm{N} / \mathrm{A}$ & 5-level EQ-5D version (EQ-5D-5L) & (135) \\
\hline 2019 & $\begin{array}{l}\text { Gàvilan-Carrera } \\
\text { et al. }\end{array}$ & CS & 70 & $\mathrm{~N} / \mathrm{A}$ & N/A & $\begin{array}{l}\text { 36-item ShortForm Health Survey } \\
\text { (SF-36) }\end{array}$ & (136) \\
\hline 2019 & Legge et al. & PR & 1683 & $\mathrm{~N} / \mathrm{A}$ & $\mathrm{N} / \mathrm{A}$ & $\begin{array}{l}\text { 36-item ShortForm Health Survey } \\
\text { (SF-36) }\end{array}$ & (137) \\
\hline 2019 & Park et al. & CC & 120 & 960 & $\begin{array}{l}\text { Systemic sclerosis } \\
(n=120), \text { rheumatoid } \\
\text { arthritis }(n=120), \text { Sjögren } \\
\text { syndrome }(n=120) \text { and } \\
600 \text { healthy controls }\end{array}$ & $\begin{array}{l}\text { 36-item ShortForm Health Survey (SF-36), } \\
\text { Short Form Six-Dimensional health index } \\
\text { (SF-6D), and EuroQol 5-dimension (EQ-5D) }\end{array}$ & (138) \\
\hline 2019 & $\begin{array}{l}\text { Conceição } \\
\text { et al. }\end{array}$ & RCT & 80 & $\mathrm{~N} / \mathrm{A}$ & $\mathrm{N} / \mathrm{A}$ & $\begin{array}{l}\text { Systemic Lupus Erythematosus-specific } \\
\text { quality of life instrument (SLEQOL) }\end{array}$ & (139) \\
\hline 2019 & $\begin{array}{l}\text { Castellano } \\
\text { Rioja et al. }\end{array}$ & CC & 161 & $\mathrm{~N} / \mathrm{A}$ & $\mathrm{N} / \mathrm{A}$ & SLE Quality of Life Questionnaire (L-QoL) & (140) \\
\hline 2019 & Delis et al. & $\mathrm{CC}$ & 196 & $\mathrm{~N} / \mathrm{A}$ & $\mathrm{N} / \mathrm{A}$ & Lupus Quality of Life (LupusQoL) & $(141)$ \\
\hline 2020 & Katz et al. & CS & 1066 & $\mathrm{~N} / \mathrm{A}$ & $N / A$ & $\begin{array}{l}\text { Lupus impact tracker (LIT), 6-item SLE- } \\
\text { Family questionnaire, and Healthy Days } \\
\text { Core Module (HDCM) }\end{array}$ & (142) \\
\hline 2020 & $\begin{array}{l}\text { Ratanasiripong } \\
\text { et al. }\end{array}$ & CS & 650 & $\mathrm{~N} / \mathrm{A}$ & $\mathrm{N} / \mathrm{A}$ & $\begin{array}{l}\text { Stress Scale (DASS), and Lupus Quality of } \\
\text { Life (LupusQoL) }\end{array}$ & (143) \\
\hline 2020 & $\begin{array}{l}\text { Louthrenoo } \\
\text { et al. }\end{array}$ & CS & 337 & $\mathrm{~N} / \mathrm{A}$ & $\mathrm{N} / \mathrm{A}$ & $\begin{array}{l}\text { 36-item ShortForm Health Survey (SF-36), } \\
\text { and Systemic Lupus Erythematosus-specific } \\
\text { quality of life instrument (SLEQOL) }\end{array}$ & (30) \\
\hline 2020 & Legge et al. & PR & 1549 & $\mathrm{~N} / \mathrm{A}$ & $N / A$ & 36-item ShortForm Health Survey (SF-36) & (144) \\
\hline
\end{tabular}

SLE, systemic lupus erythematosus; CC, case-control; CS, cross-sectional; LG, longitudinal; PR, prospective; RC, retrospective cohort; RCT, randomized clinical trial; N/A, not applicable. 
were the most commonly used tools [summarized in Table II $(6,8-12,27,145-171)]$.

\section{6-item Medical Outcome Short Form}

The SF-36 was not designed specifically for patients with SLE, but for the general population. Sixty-three studies, i.e. 53\% of the total included in our analysis, applied the SF-36. Findings from these studies showed that patients with SLE scored $<50$ for both the physical component (PCS) and the mental component scores (MCS). The same result was observed in patients affected by other autoimmune conditions considered as controls (evaluated in only three studies out of 63), while healthy controls (only analyzed in two studies out of 63) scored $>50$ for both PCS and MCS.

\section{Lupus Quality of Life}

Lupus Quality of Life (LupusQoL) is a SLE-specific HRQoL tool. It was the second most commonly applied (17 studies accounting for $14.3 \%$ of the total) and ranged from 41 to 71 .

\section{Lupus Patient-Reported Outcome}

The Lupus Patient-Reported Outcome (LupusPRO) also contains non-health related QoL (NHRQoL) domains in addition to HRQoL domains and was used in 12 studies out of 119 (10\%). In relation to HRQoL, SLE patients scored from 56 to 75 , while they scored from 59 to 72 for NHRQoL domains.

Other measures that were used in the included studies were: Lupus Impact Tracker (LIT) (6 studies =5\%), EuroQoL (EQ-5D) (6 studies $=5 \%$ ), 12-item Medical Outcome Short Form (SF-12) (5 studies $=4.2 \%)$ and Systemic Lupus Erythematosus-Specific Quality of Life Questionnaire (SLEQoL) (5 studies $=4.2 \%)$. Moreover, 75 studies out of $119(63 \%)$ used only one measure,

Table II - Summary of the quality of life measures used in the studies included in the systematic review.

\begin{tabular}{|l|c|l|c|c|c|c|}
\hline Measure & $\begin{array}{c}\text { N. of } \\
\text { items }\end{array}$ & Domains & $\begin{array}{c}\text { Questions } \\
\text { Format }\end{array}$ & $\begin{array}{c}\text { Range of } \\
\text { scoring }\end{array}$ & $\begin{array}{c}\text { N. of studies } \\
\text { that used the } \\
\text { measure }\end{array}$ & Reference \\
\hline $\begin{array}{l}\text { Lupus quality of } \\
\text { life (LupusQol) }\end{array}$ & 34 & $\begin{array}{l}\text { Physical health, emotional health, } \\
\text { body image, pain, planning, fatigue, } \\
\text { and relationships }\end{array}$ & 5 -points scale & $1-100$ & 17 & (6) \\
\hline $\begin{array}{l}\text { Problematic } \\
\text { Support Scale } \\
\text { (PSS) }\end{array}$ & 20 & $\begin{array}{l}\text { Social support, psychosocial } \\
\text { adjustment, and disease } \\
\text { characteristics }\end{array}$ & 5 -points scale & $0-60$ & 1 & (145) \\
\hline $\begin{array}{l}\text { Short-Form 36 } \\
\text { (SF-36) }\end{array}$ & 36 & $\begin{array}{l}\text { Physical functioning, bodily pain, } \\
\text { general health, vitality, social } \\
\text { functioning, role of emotional, and } \\
\text { mental health }\end{array}$ & 8-scaled scores & $1-100$ & 63 & (10) \\
\hline $\begin{array}{l}\text { Lupus patient } \\
\text { reported outcomes } \\
\text { (LupusPRO) }\end{array}$ & 44 & $\begin{array}{l}\text { Lupus symptoms, Cognition, Lupus } \\
\text { medication, Physical health, Pain- } \\
\text { vitality, Emotional health, procreation, } \\
\text { body image, general health, desires/ } \\
\text { goals, coping, social support, and } \\
\text { satisfaction with care }\end{array}$ & 6-points scale & $0-100$ & 12 & (8) \\
\hline $\begin{array}{l}\text { Pediatric Quality } \\
\text { of Life Inventory } \\
\text { version 4.0 Generic } \\
\text { Core Scales } \\
\text { (PedsQLTM 4.0) }\end{array}$ & 23 & $\begin{array}{l}\text { Physical functioning, emotional } \\
\text { functioning, social functioning, and } \\
\text { school functioning }\end{array}$ & 5-points scale & $0-100$ & 4 & (146) \\
\hline $\begin{array}{l}\text { Patient Reported } \\
\text { Outcomes } \\
\text { Measurement } \\
\text { Information } \\
\text { System (PROMIS) }\end{array}$ & 56 & $\begin{array}{l}\text { Pain, fatigue, anger, anxiety, } \\
\text { depression, physical function, } \\
\text { satisfaction with participation in } \\
\text { social roles, and satisfaction with } \\
\text { participation in discretionary social } \\
\text { activities }\end{array}$ & 5-points scale & $0-30$ & 4 & (147) \\
\hline
\end{tabular}




\begin{tabular}{|c|c|c|c|c|c|c|}
\hline Measure & $\begin{array}{l}\text { N. of } \\
\text { items }\end{array}$ & Domains & $\begin{array}{l}\text { Questions } \\
\text { Format }\end{array}$ & $\begin{array}{l}\text { Range of } \\
\text { scoring }\end{array}$ & $\begin{array}{l}\text { N. of studies } \\
\text { that used the } \\
\text { measure }\end{array}$ & Reference \\
\hline $\begin{array}{l}\text { Simple Measure of } \\
\text { Impact of Lupus } \\
\text { Erythematosus } \\
\text { in Youngsters } \\
\text { (SMILEY) }\end{array}$ & 26 & $\begin{array}{l}\text { Effects on self, limitations, social life, } \\
\text { and burden of SLE }\end{array}$ & 5-points scale & $1-100$ & 2 & (148) \\
\hline $\begin{array}{l}\text { Childhood Health } \\
\text { Assessment } \\
\text { Questionnaire } \\
\text { (C-HAQ) }\end{array}$ & 30 & $\begin{array}{l}\text { Dressing and personal care, getting } \\
\text { up, eating, walking, hygiene, reach, } \\
\text { grip, and activities }\end{array}$ & 4-points scale & $0-32$ & 3 & (149) \\
\hline $\begin{array}{l}\text { Brief Illness } \\
\text { Perception } \\
\text { Questionnaire } \\
\text { (Brief IPQ) }\end{array}$ & 9 & $\begin{array}{l}\text { Cognitive illness, emotional } \\
\text { representations, illness } \\
\text { comprehensibility, and casual } \\
\text { representation }\end{array}$ & 11-points scale & $0-100$ & 1 & (150) \\
\hline $\begin{array}{l}\text { Systemic lupus } \\
\text { erythematosus } \\
\text { observations of } \\
\text { travel burden } \\
\text { (SLEOTB) }\end{array}$ & NA & $\begin{array}{l}\text { Reliance on caregivers, meeting } \\
\text { financial priorities, and pain and } \\
\text { physical limitations }\end{array}$ & NA & NA & 1 & (151) \\
\hline $\begin{array}{l}\text { Visual analogue } \\
\text { scale (VAS) }\end{array}$ & NA & NA & NA & $0-100$ & 3 & (152) \\
\hline $\begin{array}{l}\text { Krupp's fatigue } \\
\text { severity scale } \\
\text { (FSS) }\end{array}$ & 20 & NA & 7-points scale & $0-60$ & 7 & (27) \\
\hline $\begin{array}{l}\text { Functional } \\
\text { Assessment of } \\
\text { Chronic Illness } \\
\text { Therapy-Fatigue } \\
\text { (FACIT-F) }\end{array}$ & 27 & $\begin{array}{l}\text { Physical well-being, Social/Family } \\
\text { well-being, Emotional well-being, and } \\
\text { Functional well-being }\end{array}$ & 5-points scale & $0-100$ & 9 & (12) \\
\hline $\begin{array}{l}\text { Lupus Impact } \\
\text { Tracker (LIT) }\end{array}$ & 21 & $\begin{array}{l}\text { Cognition, lupus medications, } \\
\text { physical health, pain/fatigue impact, } \\
\text { emotional health, body image, and } \\
\text { planning/desires/goals }\end{array}$ & $\begin{array}{l}\text { 100-points } \\
\text { scale }\end{array}$ & $0-100$ & 6 & (9) \\
\hline $\begin{array}{l}\text { Short Form } \\
\text { health survey } \\
\text { questionnaire } \\
\text { (SF-12) }\end{array}$ & 12 & $\begin{array}{l}\text { Physical functioning, role physical, } \\
\text { bodily pain, general health, vitality, } \\
\text { social functioning, role emotional, and } \\
\text { mental health }\end{array}$ & 8-points scale & $1-100$ & 5 & (11) \\
\hline $\begin{array}{l}\text { Multidimensional } \\
\text { assessment of } \\
\text { fatigue (MAF) } \\
\text { scale }\end{array}$ & 16 & NA & 10 -points scale & $0-150$ & 2 & (153) \\
\hline EuroQol (EQ-5D) & 5 & $\begin{array}{l}\text { Mobility, Self-Care, Usual activity, } \\
\text { Pain/Discomfort, and Anxiety/ } \\
\text { Depression }\end{array}$ & 5 levels & $0-100$ & 6 & (154) \\
\hline $\begin{array}{l}\text { Neuro-QoL } \\
\text { (Quality of Life } \\
\text { in Neurological } \\
\text { Disorders) }\end{array}$ & 13 & $\begin{array}{l}\text { Upper extremity function and applied } \\
\text { cognition }\end{array}$ & 5-points scale & $0-80$ & 1 & (155) \\
\hline $\begin{array}{l}\text { Pittsburgh Sleep } \\
\text { Quality Index } \\
\text { (PSQI) }\end{array}$ & 19 & $\begin{array}{l}\text { Sleep quality, sleep latency, sleep } \\
\text { duration, habitual sleep efficiency, } \\
\text { sleep disturbances, use of sleeping } \\
\text { medication, and daytime dysfunction }\end{array}$ & $\begin{array}{l}7 \text { component } \\
\text { scores }\end{array}$ & $0-21$ & 1 & (156) \\
\hline
\end{tabular}




\begin{tabular}{|c|c|c|c|c|c|c|}
\hline Measure & $\begin{array}{l}\text { N. of } \\
\text { items }\end{array}$ & Domains & $\begin{array}{l}\text { Questions } \\
\text { Format }\end{array}$ & $\begin{array}{l}\text { Range of } \\
\text { scoring }\end{array}$ & $\begin{array}{l}\text { N. of studies } \\
\text { that used the } \\
\text { measure }\end{array}$ & Reference \\
\hline $\begin{array}{l}\text { Self-developed } \\
\text { questionnaire }\end{array}$ & 30 & $\begin{array}{l}\text { Route to diagnosis, therapy, social } \\
\text { environment, information, and } \\
\text { communication, and demographic } \\
\text { data }\end{array}$ & NA & NA & 1 & $\begin{array}{l}\text { www. } \\
\text { lebenmitlupus. } \\
\text { org }\end{array}$ \\
\hline $\begin{array}{l}\text { Health Assessment } \\
\text { Questionnaire } \\
\text { (HAQ) }\end{array}$ & 20 & $\begin{array}{l}\text { Dressing and personal care, getting } \\
\text { up, eating, walking, hygiene, reach, } \\
\text { grip, and activities }\end{array}$ & 4-points scale & $0-32$ & 3 & (157) \\
\hline $\begin{array}{l}\text { Hospital Anxiety } \\
\text { and Depression } \\
\text { Scale (HADS) }\end{array}$ & 14 & Mood and anxiety & 4-points scale & $0-100$ & 2 & (158) \\
\hline $\begin{array}{l}\text { Perceived Stress } \\
\text { Scale (PSS) }\end{array}$ & 10 & $\begin{array}{l}\text { Family pressure, academic pressure, } \\
\text { interpersonal conflicts, physical } \\
\text { health problems, and others }\end{array}$ & 5-points scale & $0-40$ & 1 & (159) \\
\hline $\begin{array}{l}\text { Nottingham Health } \\
\text { Profile (NHP) }\end{array}$ & 38 & $\begin{array}{l}\text { Physical mobility, social isolation, } \\
\text { emotional reactions, pain, sleep, and } \\
\text { energy }\end{array}$ & 7-points scale & $0-100$ & 1 & (160) \\
\hline $\begin{array}{l}\text { Oral health impact } \\
\text { profile (OHIP-49) }\end{array}$ & 49 & $\begin{array}{l}\text { Functional limitation, physical pain, } \\
\text { psychological discomfort, physical } \\
\text { disability, psychological disability, } \\
\text { social disability, and handicap }\end{array}$ & 5-points scale & $0-70$ & 1 & (161) \\
\hline $\begin{array}{l}\text { Insomnia Severity } \\
\text { Index }\end{array}$ & 7 & NA & 5-points scale & $0-49$ & 1 & (162) \\
\hline $\begin{array}{l}\text { Patient Health } \\
\text { Questionnarie-9 }\end{array}$ & 9 & NA & 5-points scale & $0-27$ & 2 & (163) \\
\hline $\begin{array}{l}\text { Systemic Lupus } \\
\text { Erythematosus- } \\
\text { Specific Quality of } \\
\text { Life Questionnaire } \\
\text { (SLEQOL) }\end{array}$ & 40 & $\begin{array}{l}\text { Physical functioning, activities, } \\
\text { symptoms, treatment, mood, and } \\
\text { self-image }\end{array}$ & 7-points scale & $40-280$ & 5 & (164) \\
\hline $\begin{array}{l}\text { 6-item SLE-Family } \\
\text { questionnaire }\end{array}$ & 6 & $\begin{array}{l}\text { Fatigue, activity participation, mental } \\
\text { health, isolation, love and intimacy, } \\
\text { and family roles }\end{array}$ & 6 questions & $0-10$ & 1 & (165) \\
\hline $\begin{array}{l}\text { Healthy Days Core } \\
\text { Module (HDCM) }\end{array}$ & 4 & NA & 4 questions & $0-30$ & 1 & (166) \\
\hline $\begin{array}{l}\text { Generalized } \\
\text { Anxiety Disorder } 7 \\
\text { (GAD7) }\end{array}$ & 7 & Anxiety & 4-points scale & $0-21$ & 1 & (167) \\
\hline $\begin{array}{l}\text { Short Form Six- } \\
\text { Dimensional health } \\
\text { index (SF-6D) }\end{array}$ & 11 & $\begin{array}{l}\text { Physical functioning, role limitation, } \\
\text { social functioning, pain, mental } \\
\text { health, and vitality }\end{array}$ & $\begin{array}{l}\text { Continuous } \\
\text { measure }\end{array}$ & $0-100$ & 1 & (168) \\
\hline $\begin{array}{l}\text { Lupus } \\
\text { Erythematosus } \\
\text { Quality of Life } \\
\text { Questionnaire: } \\
\text { CVLEC }\end{array}$ & 36 & $\begin{array}{l}\text { Functioning, emotions, symptoms, } \\
\text { body image, and photosensitivity }\end{array}$ & 5-points scale & $36-180$ & 1 & (169) \\
\hline $\begin{array}{l}\text { Overactive bladder } \\
\text { questionnaire short } \\
\text { form (OABq-SF) }\end{array}$ & 19 & $\begin{array}{l}\text { Symptom bother and health-related } \\
\text { quality of life }\end{array}$ & 6-points scale & $0-100$ & 1 & (170) \\
\hline $\begin{array}{l}\text { Chalder Fatigue } \\
\text { Scale (CFS) }\end{array}$ & 11 & Physical and mental & 4-points scale & $0-100$ & 1 & (171) \\
\hline
\end{tabular}


31 out of $119(26 \%)$ used a combination of two measures, 11 out of $119(9.25 \%)$ used a combination of three measures and 2 out of $119(1.7 \%)$ used more than four measures.

\section{DISCUSSION}

HRQoL tools are widely used in many studies of SLE patients, from randomized controlled studies to patient management strategy studies, thus gaining progressively more interest in the research community.

As to the overall results of our systematic review, SF-36 was the most widely used generic tool to measure HRQoL in SLE (16). Although SF-36 is diverse, well-validated, and sensitive to change (17), its generic nature may not permit to assess specific dimensions of HRQoL in SLE patients, such as physical appearance and intimate relationships (6). Furthermore, SF-36 does not include the assessment of organspecific aspects of HRQoL, such as skin or kidney-related complications. Although SF-36 is sensitive to change over time, it might sometimes miss meaningful changes noted in longitudinal studies or trials (18), thus proving more suitable for studies with cross-sectional design.

The LupusQoL is a disease-specific HRQoL tool that was developed and validated in 2007 at the Rheumatology department of Blackburn Royal Infirmary. Four domains of LupusQoL are similar to SF-36, namely emotional health, physical health, pain, and social role. Both LupusQoL and SF-36 can be used to reflect improvement and worsening (19), however the disease-related LupusQoL is considered the most appropriate validated tool for cross-sectional evaluations of HRQoL in SLE (7). Few longitudinal studies in our analysis used the LupusQoL (20-22). Although these studies showed fair sensitivity to change, some domains, such as the body image domain, were irresponsive to change, especially in relation to dermatological or musculoskeletal flares, or treatment (20), It has been previously shown that patients with a more active disease report poorer HRQoL across all LupusQoL domains, ex- cept for fatigue (7). This suggests that the use of another HRQoL tool in combination with LupusQoL could be a better approach to assess fatigue (23). Indeed, many tools can be used to assess fatigue among SLE patients. For example, the functional assessment of chronic illness therapy-fatigue (FACIT-F) explores self-reported aspects of physical and mental fatigue and their effects on function and daily living (24). A strong positive correlation was observed between FACIT-F domains and many SF36 domains, with a similar impact of fatigue on both physical and mental health (25). Along with FACIT-F and visual analogue scale (VAS), the Krupp's fatigue severity scale (FSS) is a frequently used instrument to measure fatigue among patients with SLE (26). FSS has good psychometric properties and was validated in multiple languages. Additionally, it was designed to measure the outcome of fatigue on functional performance such as exercise, motivation, and daily activities (27). Although both the FACIT-F scale and the FSS have good construct validity, when assessing fatigue in SLE patients (28), FSS was reported to have inferior internal consistency and sensitivity than the FACIT-F scale (29). Other less commonly used fatigue assessment tools, such as the multidimensional assessment of fatigue (MAF) scale and Chalder fatigue scale (CFS), were also less studied in SLE, particularly in a prospective fashion, as evidenced in our analysis.

Certain domains of the lupus experience, such as self-image, family planning, or impact of medication, can be neglected by some SLE QoL assessment tools. To address these gaps, the Lupus Patient-Reported Outcome (LupusPRO) was developed in 2012. This tool contains HRQoL domains, such as lupus symptomatology, physical health, and body image, as well as nonHRQoL domains such as desires/goals and satisfaction with care. LupusPRO demonstrates good internal consistency and good test reliability. While this tool is sensitive to longitudinal changes and widely-validated, there are still no data available on its responsiveness using the standardized 
response means (8). Similar to SF-36, LupusPRO reports greater magnitude of change for improvement than for deterioration (6). However, LupusPRO can better detect organ-specific effects on QoL (8).

Despite all the data considered, it is sometimes difficult to appropriately compare different domains of different tools. For example, SF-36 and LupusQoL have strong correlations among physical health/physical functioning, emotional health/mental health, pain/bodily pain, and fatigue/vitality domains (6). Similarly, SLEQOL and SF-36 correlate well in physical functioning, activities, and symptoms scores (30). However, many aspects, possibly affecting HRQoL in SLE patients and not assessed by certain tools, can affect physical functioning, thus leading to a difference in sensitivity between the tools. Therefore, future clinical trials should use more than one tool to fully capture the experience of patients with SLE and predict which factors can affect which domains.

Our analysis also included several measures of pediatric SLE. These tools vary in their ability to predict change in disease activity over time. The Childhood Health Assessment Questionnaire, used in 2 studies $(1.68 \%)$ in our analysis, is adequate for assessing physical function and disability in pediatric SLE, but its primary emphasis is on SLE symptoms related to arthritis (31). Among all the measures, the Simple Measure of Impact of Lupus Erythematosus in Youngsters (SMILEY), used in 2 studies $(1.68 \%)$, is the only disease specific multidimensional measure (31). On the contrary, the Pediatric Quality of Life Inventory (Ped$\mathrm{sQL}$ ), used in 3 studies (2.5\%), does not correlate significantly with disease severity, self-concept and socioeconomic status (32), implying that even though the system-specific module encompasses appropriate domains, pediatric SLE patients may require additional items to completely explain the effect of the disease on their QoL.

We acknowledge that this study has some limitations. Although our initial search strategy aimed at identifying all papers relevant to our objectives, it is possible that some studies may not have been retrieved, due to the wide heterogeneity of keywording in this field. Also, aggregation of studies that differ in objectives, terms of intervention or patient type and HRQoL tools used could have led to the generalization of study findings. For instance, the diverse study designs led to the inclusion of both healthy controls and controls affected by other chronic autoimmune conditions and to a disproportionate amount of SLE patients and controls. Moreover, since most studies did not report patients' clinical manifestations, it was not possible to distinguish between different subclasses of SLE. Finally, there are some instruments for cutaneous lupus which could be relevant in patients with SLE who had skin manifestations, but the evaluation of these instruments was beyond the aim of this analysis.

\section{CONCLUSIONS}

Based on this systematic review of the literature, quality of life in patients with SLE appears to be poor and overall lower when compared to both the general population and patients with other chronic conditions. Furthermore, some of the tools currently used to measure quality of life (e.g., SF36) were not specifically developed for the SLE population, and, therefore, may not be capturing all the relevant domains, especially in longitudinal studies. Some others, like the LupusQoL, while specifically developed in patients with SLE, lack prospective validation investigating performances in different geographical settings. SLE specific tools validated prospectively with the ability to detect organ-specific effects on HRQoL, such as LupusPRO, can be occasionally insensitive to treatment-related changes. Therefore, future qualitative studies are needed to improve the understanding of the disease burden on quality of life from the patient's perspective. Similarly, both SLE-specific and not-SLE specifics measures should be included systemically in future prospective studies to investigate comparability and ensure that the universe of items relevant to SLE patients is properly captured. 


\section{Acknowledgments}

None

\section{Authorship Contributions}

$\mathrm{MR}, \mathrm{GEH}, \mathrm{AB}$ searched the literature, assisted with the organization of the manuscript, interpreted and collected data, and wrote and edited the review. DR, IU, AT and SS interpreted and collected data, helped to design the figures and panel, and wrote and edited the review.

\section{Declaration of Interest}

None

\section{Ethics approval and consent to partici- pate}

None

\section{Consent for publication}

None

\section{Availability of data and materials \\ None}

\section{Funding}

None

\section{REFERENCES}

1. Tsokos GC. Mechanisms of disease: systemic lupus erythematosus. N Engl J Med. 2011; 365: 2110-21.

2. Kaul A, Gordon C, Crow MK, et al. Systemic lupus erythematosus. Nat Rev Dis Prim. 2016; 2: 16039. Available from: www.nature.com/ nrdp

3. Kernder A, Elefante E, Chehab G, et al. The patient's perspective: Are quality of life and disease burden a possible treatment target in systemic lupus erythematosus? Rheumatol (United Kingdom). 2020; 59: V63-8.

4. Almehed K, Carlsten H, Forsblad-D'Elia H. Health-related quality of life in systemic lupus erythematosus and its association with disease and work disability. Scand J Rheumatol. 2010; 39: 58-62.

5. Elera-Fitzcarrald C, Fuentes A, González LA, et al. Factors affecting quality of life in patients with systemic lupus erythematosus: important considerations and potential interventions. Expert Rev Clin Immunol. 2018; 14: 915-31.

6. McElhone K, Abbott J, Shelmerdine J, et al. Development and validation of a diseasespecific health-related quality of life measure, the LupusQoL, for adults with systemic lupus erythematosus. Arthritis Care Res. 2007; 57: 972-9.

7. Yazdany J. Health-related quality of life measurement in adult systemic lupus erythematosus: Lupus Quality of Life (LupusQoL), Systemic Lupus Erythematosus-Specific Quality of Life Questionnaire (SLEQOL), and Systemic Lupus Erythematosus Quality of Life Questionnaire (L-QoL). Arthritis Care Res. 2011; 63.

8. Jolly M, Pickard AS, Block JA, et al. Diseasespecific patient reported outcome tools for systemic lupus erythematosus. Semin Arthritis Rheum. 2012; 42: 56-65.

9. Jolly M, Garris CP, Mikolaitis RA, et al. Development and validation of the lupus impact tracker: a patient-completed tool for clinical practice to assess and monitor the impact of systemic lupus erythematosus. Arthritis Care Res. 2014; 66: 1542-50.

10. Ware JE, Ware J. SF-36 Physical and Mental Health Summary Scales: a User's Manual Estimation of medical care total expenditures. View Project 1993 (cited 2021 Feb 16). Available from: https://www.researchgate.net/publication/292390260

11. Ware JE, Kosinski M, Keller SD. A 12-item short-form health survey: construction of scales and preliminary tests of reliability and validity. Med Care. 1996; 34: 220-33.

12. Webster K, Cella D, Yost K. The Functional Assessment of Chronic Illness Therapy (FACIT) Measurement System: Properties, applications, and interpretation (Internet). Health Qual Life Outcomes. 2003; 1: 79.

13. Jolly M, Garris CP, Mikolaitis RA, et al. Development and validation of the lupus impact tracker: A patient-completed tool for clinical practice to assess and monitor the impact of systemic lupus erythematosus. Arthritis Care Res. 2014; 66: 1542-50.

14. Yazdany J. Health-related quality of life measurement in adult systemic lupus erythematosus: Lupus Quality of Life (LupusQoL), Systemic Lupus Erythematosus-Specific Quality of Life Questionnaire (SLEQOL), and Systemic Lupus Erythematosus Quality of Life Questionnaire (L-QoL). Arthritis Care Res. 2011; 63 (Suppl. 11).

15. PRISMA 2009 Checklist. Available from: http://prisma-statement.org/documents/PRISMA\%202009\%20checklist.pdf

16. Stoll T, Gordon C, Seifert B, et al. Consistency and validity of patient administered assessment of quality of life by the MOS SF-36; Its association with disease activity and damage in patients with systemic lupus erythematosus. J Rheumatol. 1997; 24: 1608-14.

17. Touma Z, Gladman DD, Ibanẽz D, Urowitz MB. Is there an advantage over SF-36 with a quality of life measure that is specific to 
systemic lupus erythematosus? J Rheumatol. 2011; 38: 1898-905.

18. Leong KP, Kong KO, Thong BYH, et al. Development and preliminary validation of a systemic lupus erythematosus-specific qualityof-life instrument (SLEQOL). Rheumatology. 2005; 44: 1267-76.

19. Nantes SG, Strand V, Su J, Touma Z. Comparison of the sensitivity to change of the 36 -item short form health survey and the lupus quality of life measure using various definitions of minimum clinically important differences in patients with active systemic lupus erythematosus. Arthritis Care Res. 2018; 70: 125-33.

20. Corneloup M, Maurier F, Wahl D, et al. Disease-specific quality of life following a flare in systemic lupus erythematosus: an item response theory analysis of the French EQUAL cohort. Rheumatol (United Kingdom). 2020; 59: 1398-406.

21. Devilliers H, Bonithon-Kopp C, Jolly M. The lupus impact tracker is responsive to changes in clinical activity measured by the systemic lupus erythematosus responder index. Lupus. 2017; 26: 396-402.

22. McElhone K, Abbott J, Sutton C, et al. Sensitivity to change and minimal important differences of the LupusQoL in patients with systemic lupus erythematosus. Arthritis Care Res. 2016; 68: 1505-13.

23. Cosatti MA, Muñoz S, Alba P, et al. Multicenter study to assess presenteeism in systemic lupus erythematosus and its relationship with clinical and sociodemographic features. Lupus. 2018; 27: 33-9.

24. Yellen SB, Cella DF, Webster K, et al. Measuring fatigue and other anemia-related symptoms with the Functional Assessment of Cancer Therapy (FACT) measurement system. J Pain Symptom Manage. 1997; 13: 63-74.

25. Elefante E, Tani C, Stagnaro C, et al. Impact of fatigue on health-related quality of life and illness perception in a monocentric cohort of patients with systemic lupus erythematosus. BMJ Open Sport Exerc Med. 2020; 6(1).

26. Barbacki A, Petri M, Avina-Zubieta A, et al. Fatigue measurements in systemic lupus erythematosus. J Rheumatol. 2019; 46: 1470-7.

27. Krupp LB, Larocca NG, Muir Nash J, Steinberg AD. The fatigue severity scale: Application to patients with multiple sclerosis and systemic lupus erythematosus. Arch Neurol. 1989; 46: 1121-3.

28. Kosinski M, Gajria K, Fernandes AW, Cella D. Qualitative validation of the FACIT-Fatigue scale in systemic lupus erythematosus. Lupus. 2013; 22: 422-30.

29. Goligher E, Pouchot J, Brant R, et al. Minimal clinically important difference for 7 measures of fatigue in patients with systemic lupus erythematosus. J Rheumatol. 2008; 35.
30. Louthrenoo W, Kasitanon N, Morand E, et al. Comparison of performance of specific (SLEQOL) and generic (SF36) health-related quality of life questionnaires and their associations with disease status of systemic lupus erythematosus: A longitudinal study. Arthritis Res Ther. 2020; 22(1).

31. Hersh A. Measures of health-related quality of life in pediatric systemic lupus erythematosus: Childhood Health Assessment Questionnaire (C-HAQ), Child Health Questionnaire (CHQ), Pediatric Quality of Life Inventory Generic Core Module (PedsQL-GC), Pediatric Quality of Life Inventory Rheumatology Module (PedsQL-RM), and Simple Measure of Impact of Lupus Erythematosus in Youngsters (SMILEY). Arthritis Care Res. 2011; 63 (Suppl. 11).

32. Moorthy LN, Baldino ME, Kurra V, et al. Relationship between health-related quality of life, disease activity and disease damage in a prospective international multicenter cohort of childhood onset systemic lupus erythematosus patients. Lupus. 2017; 26: 255-65.

33. Mirbagher L, Gholamrezaei A, Hosseini N, Sayed Bonakdar Z. Sleep quality in women with systemic lupus erythematosus: Contributing factors and effects on health-related quality of life. Int J Rheum Dis. 2016; 19: 305-11.

34. Mazzoni D, Cicognani E. Positive and problematic support, stress and quality of life in patients with systemic lupus erythematosus. Anxiety Stress Coping. 2016; 29: 542-51.

35. Yilmaz-Oner S, Oner C, Dogukan FM, et al. Health-related quality of life assessed by LupusQoL questionnaire and SF-36 in Turkish patients with systemic lupus erythematosus. Clin Rheumatol. 2016; 35: 617-22.

36. Wallace DJ, Hobbs K, Clowse MEB, et al. Long-term safety and efficacy of epratuzumab in the treatment of moderate-to- severe systemic lupus erythematosus: results from an open-label extension study. Arthritis Care Res. 2016; 68: 534-43.

37. Mazzoni D, Cicognani E. The problematic support scale: a validation among patients with systemic lupus erythematosus. J Health Psychol. 2016; 21: 1711-7.

38. Hanly JG, O'Keeffe AG, Su L, et al. The frequency and outcome of lupus nephritis: Results from an international inception cohort study. Rheumatol (United Kingdom). 2015; 55: 252-62.

39. Boström C, Elfving B, Dupré B, et al. Effects of a one-year physical activity programme for women with systemic lupus erythematosus A randomized controlled study. Lupus. 2016; 25: 602-16.

40. Fidler L, Keen KJ, Touma Z, Mittoo S. Impact of pulmonary disease on patient-reported outcomes and patient-performed functional test- 
ing in systemic lupus erythematosus. Lupus. 2016; 25: 1004-11.

41. Mahieu MA, Ahn GE, Chmiel JS, et al. Fatigue, patient reported outcomes, and objective measurement of physical activity in systemic lupus erythematosus. Lupus. 2016; 25 : 1190-9.

42. Jolly M, Galicier L, Aumaître O, et al. Quality of life in systemic lupus erythematosus: Description in a cohort of French patients and association with blood hydroxychloroquine levels. Lupus. 2016; 25: 735-40.

43. Wang H, Guo X, Lai J, et al. Predictors of health-related quality of life in patients with systemic lupus erythematosus associated pulmonary arterial hypertension. Clin Exp Rheumatol. 2016; 34: 291-5.

44. Macêdo EA, Appenzeller S, Costallat LTL. Gender differences in systemic lupus erythematosus concerning anxiety, depression and quality of life. Lupus. 2016; 25: 1315-27.

45. Otter SJ, Kumar S, Gow P, et al. Patterns of foot complaints in systemic lupus erythematosus: A cross sectional survey. J Foot Ankle Res. 2016; 9: 4-11.

46. Oliveira-Santos M, Verani JF de S, Camacho LAB, et al. Effectiveness of pharmaceutical care for drug treatment adherence in patients with systemic lupus erythematosus in Rio de Janeiro, Brazil: Study protocol for a randomized controlled trial. Trials. 2016; 17(1).

47. Mok C, Chan KL, Ho LY. Association of depressive/anxiety symptoms with quality of life and work ability in patients with systemic lupus erythematosus. Clin Exp Rheumatol. 2016; 34: 389-95.

48. Legge A, Doucette S, Hanly JG. Predictors of organ damage progression and effect on healthrelated quality of life in systemic lupus erythematosus. J Rheumatol. 2016; 43: 1050-6.

49. Ramondt S, Tiemensma J, Cameron LD, et al. Drawings of blood cells reveal people's perception of their blood disorder: A pilot study. PLoS One. 2016; 11: 1-11.

50. Mazzoni D, Cicognani E, Prati G. Health-related quality of life in systemic lupus erythematosus: A longitudinal study on the impact of problematic support and self-efficacy. Lupus. 2017; 26: 125-31.

51. Sebastiani GD, Prevete I, Iuliano A, Minisola G. The importance of an early diagnosis in systemic lupus erythematosus. Isr Med Assoc J. 2016; 18: 212-5.

52. Piga M, Gabba A, Congia M, et al. Predictors of musculoskeletal flares and Jaccoud's arthropathy in patients with systemic lupus erythematosus: a 5-year prospective study. Semin Arthritis Rheum. 2016; 46: 217-24.

53. Williams EM, Lorig K, Glover S, et al. Intervention to Improve Quality of life for AfricanAmerican lupus patients (IQAN): Study pro- tocol for a randomized controlled trial of a unique a la carte intervention approach to selfmanagement of lupus in African Americans. BMC Health Serv Res. 2016; 16: 1-13.

54. López-Pina JA, Meseguer-Henarejos AB, Gascón-Cánovas JJ, et al. Measurement properties of the brief resilient coping scale in patients with systemic lupus erythematosus using rasch analysis. Health Qual Life Outcomes. 2016; 14: 1-8.

55. Pinto AJ, Miyake CNH, Benatti FB, et al. Reduced aerobic capacity and quality of life in physically inactive patients with systemic lupus erythematosus with mild or inactive disease. Arthritis Care Res. 2016; 68: 1780-6.

56. Machado Escobar MA, Yacuzzi MS, Martinez $\mathrm{RN}$, et al. Validation of an Argentine version of lupus quality of life questionnaire. Lupus. 2016; 25: 1615-22.

57. Chen HH, Chen DY, Chen YM, Lai KL. Health-related quality of life and utility: comparison of ankylosing spondylitis, rheumatoid arthritis, and systemic lupus erythematosus patients in Taiwan. Clin Rheumatol. 2017; 36: 133-42.

58. Inoue M, Shiozawa K, Yoshihara R, et al. The Japanese LupusPRO: A cross-cultural validation of an outcome measure for lupus. Lupus. 2017; 26: 849-56.

59. Jones JT, Carle AC, Wootton J, et al. Validation of patient-reported outcomes measurement information system short forms for use in childhood-onset systemic lupus erythematosus. Arthritis Care Res. 2017; 69: 133-42.

60. Antony A, Kandane-Rathnayake RK, Ko T, et al. Validation of the lupus impact tracker in an Australian patient cohort. Lupus. 2017; 26: 98-105.

61. Brandt JE, Drenkard C, Kan H, et al. External Validation of the lupus impact tracker in a Southeastern US longitudinal cohort with systemic lupus erythematosus. Arthritis Care Res. 2017; 69: 842-8.

62. Yilmaz-Oner S, Ilhan B, Can M, et al. Fatigue in systemic lupus erythematosus: Association with disease activity, quality of life and psychosocial factors. Z Rheumatol. 2017; 76: 913-9.

63. Calderón J, Flores P, Aguirre JM, et al. Impact of cognitive impairment, depression, disease activity, and disease damage on quality of life in women with systemic lupus erythematosus. Scand J Rheumatol. 2017; 46: 273-80.

64. Lai JS, Beaumont JL, Jensen SE, et al. An evaluation of health-related quality of life in patients with systemic lupus erythematosus using PROMIS and Neuro-QoL. Clin Rheumatol. 2017; 36: 555-62.

65. Azizoddin DR, Olmstead R, Cost C, et al. A multi-group confirmatory factor analyses of the LupusPRO between southern California and 
Filipino samples of patients with systemic lupus erythematosus. Lupus. 2017; 26: 967-74.

66. Etchegaray-Morales I, Méndez-Martínez S, Jiménez-Hernández C, et al. Factors associated with health-related quality of life in Mexican lupus patients using the LupusQoL. PLoS One. 2017; 12: 1-10.

67. Inoue M, Shiozawa K, Yoshihara R, et al. Predictors of poor sleep quality in patients with systemic lupus erythematosus. Clin Rheumatol. 2017; 36: 1053-62.

68. Schneider M, Mosca M, Pego-Reigosa JM, et al. Cross-cultural validation of lupus impact tracker in five European clinical practice settings. Rheumatol (United Kingdom). 2017; 56: 818-28.

69. Stummvoll G, Stamm T. The patients' perspective: living with lupus in Austria. Wien Klin Wochenschr. 2017; 129: 593-7.

70. Chaigne B, Finckh A, Alpizar-Rodriguez D, et al. Differential impact of systemic lupus erythematosus and rheumatoid arthritis on health-related quality of life. Qual Life Res. 2017; 26: 1767-75.

71. Chiara T, Linda C, Andrea DS, et al. Impact of joint involvement on patients reported outcomes in systemic lupus erythematosus. Curr Rheumatol Rev. 2017; 14: 188-92.

72. Golder V, Kandane-Rathnayake R, Hoi AYB, et al. Association of the lupus low disease activity state (LLDAS) with health-related quality of life in a multinational prospective study. Arthritis Res Ther. 2017; 19: 1-11.

73. Chaigne B, Chizzolini C, Perneger T, et al. Impact of disease activity on health-related quality of life in systemic lupus erythematosus - a cross-sectional analysis of the Swiss Systemic Lupus Erythematosus Cohort Study (SSCS). BMC Immunol. 2017; 18: 1-9.

74. Magro-Checa C, Beaart-Van De Voorde LJJ, Middelkoop HAM, et al. Outcomes of neuropsychiatric events in systemic lupus erythematosus based on clinical phenotypes; Prospective data from the Leiden NP SLE cohort. Lupus. 2017; 26: 543-51.

75. Kent T, Davidson A, Newman D, et al. Burden of illness in systemic lupus erythematosus: Results from a UK patient and carer online survey. Lupus. 2017; 26: 1095-100.

76. Kasturi S, Szymonifka J, Burket JC, et al. Validity and reliability of patient reported outcomes measurement information system computerized adaptive tests in systemic lupus erythematosus. J Rheumatol. 2017; 44: 1024-31.

77. Monahan RC, Beaart-Van De Voorde LJJ, Steup-Beekman GM, et al. Neuropsychiatric symptoms in systemic lupus erythematosus: Impact on quality of life. Lupus. 2017; 26: 1252-9.

78. Mok CC, Ho LY, Tse SM, Chan KL. Prevalence of remission and its effect on damage and quality of life in Chinese patients with systemic lupus erythematosus. Ann Rheum Dis. 2017; 76: 1420-5.

79. Uzuner S, Sahin S, Durcan G, et al. The impact of peer victimization and psychological symptoms on quality of life in children and adolescents with systemic lupus erythematosus. Clin Rheumatol. 2017; 36: 1297-304.

80. Meseguer-Henarejos AB, Gascón-Cánovas JJ, López-Pina JA. Components of quality of life in a sample of patients with lupus: a confirmatory factor analysis and Rasch modeling of the LupusQoL. Clin Rheumatol. 2017; 36: 1789-95.

81. Duruöz MT, Unal C, Toprak CS, et al. The validity and reliability of systemic lupus erythematosus quality of life questionnaire (L-QoL) in a Turkish population. Lupus. 2017; 26: 1528-33.

82. Shamekhi Z, Amani R, Habibagahi Z, et al. A randomized, double-blind, placebo-controlled clinical trial examining the effects of green tea extract on systemic lupus erythematosus disease activity and quality of life. Phyther Res. 2017; 31: 1063-71.

83. Corrêa JD, Branco LGA, Calderaro DC, et al. Impact of systemic lupus erythematosus on oral health-related quality of life. Lupus. 2018; 27: 283-9.

84. Carnero Contentti E, Genco ND, Hryb JP, et al. Impact of multiple sclerosis on quality of life: Comparison with systemic lupus erythematosus. Clin Neurol Neurosurg. 2017; 163 : 149-55.

85. Margiotta DPE, Basta F, Dolcini G, et al. The relation between, metabolic syndrome and quality of life in patients with systemic lupus erythematosus. PLoS One. 2017; 12: 1-17.

86. Muhammed H, Goyal M, Lal V, et al. Neuropsychiatric manifestations are not uncommon in Indian lupus patients and negatively affect quality of life. Lupus. 2018; 27: 688-93.

87. Hersh AO, Case SM, Son MB. Predictors of disability in a childhood-onset systemic lupus erythematosus cohort: results from the CARRA Legacy Registry. Lupus. 2018; 27: 494-500.

88. Piga M, Congia M, Gabba A, et al. Musculoskeletal manifestations as determinants of quality of life impairment in patients with systemic lupus erythematosus. Lupus. 2018; 27: 190-8.

89. Iudici M, Pantano I, Fasano S, et al. Health status and concomitant prescription of immunosuppressants are risk factors for hydroxychloroquine non-adherence in systemic lupus patients with prolonged inactive disease. Lupus. 2018; 27: 265-72.

90. Donnelly C, Cunningham N, Jones JT, et al. Fatigue and depression predict reduced health-related quality of life in childhoodonset lupus. Lupus. 2018; 27: 124-33. 
91. Zeng F, Xu Q, Liu D, et al. Relatives' quality of life and psychological disturbance: A new concern of SLE management. Clin Rheumatol. 2018; 37: 67-73.

92. Jolly M, Toloza S, Goker B, et al. Diseasespecific quality of life in patients with lupus nephritis. Lupus. 2018; 27: 257-64.

93. Golder V, Ooi JJY, Antony AS, Ko T, et al. Discordance of patient and physician health status concerns in systemic lupus erythematosus. Lupus. 2018; 27: 501-6.

94. Baba S, Katsumata Y, Okamoto Y, et al. Reliability of the SF-36 in Japanese patients with systemic lupus erythematosus and its associations with disease activity and damage: a two-consecutive year prospective study. Lupus. 2018; 27: 407-16.

95. Lee JW, Kang JH, Lee KE, et al. Effects of risk factors for and components of metabolic syndrome on the quality of life of patients with systemic lupus erythematosus: a structural equation modeling approach. Qual Life Res. 2018; 27: 105-13.

96. Azizoddin DR, Weinberg S, Gandhi N, et al. Validation of the LupusPRO version 1.8: an update to a disease-specific patient-reported outcome tool for systemic lupus erythematosus. Lupus. 2018; 27: 728-37.

97. Jiang HZ, Lin ZG, Li HJ, et al. The Chinese version of the SLEQOL is a reliable assessment of health-related quality of life in han chinese patients with systemic lupus erythematosus. Clin Rheumatol. 2018; 37: 151-60.

98. Elera-Fitzcarrald C, Alva M, GamboaCardenas R, et al. Factors associated with health-related quality of life in Peruvian patients with systemic lupus erythematosus. Lupus. 2018; 27: 913-9.

99. Azizoddin DR, Gandhi N, Weinberg S, et al. Fatigue in systemic lupus: the role of disease activity and its correlates. Lupus. 2019; 28: 163-73.

100. Tsang-A-Sjoe MWP, Bultink IEM, Heslinga $\mathrm{M}$, et al. The relationship between remission and health-related quality of life in a cohort of SLE patients. Rheumatol (United Kingdom). 2019; 58: 628-35.

101. Pereira e Silva R, Romão VC, Neves M, et al. Overactive bladder symptom bother and health-related quality of life in patients with systemic lupus erythematosus and primary Sjögren syndrome. Lupus. 2019; 28: 27-33.

102. Strand V, Berry P, Lin X, et al. Long-term impact of belimumab on health-related quality of life and fatigue in patients with systemic lupus erythematosus: six years of treatment. Arthritis Care Res. 2019; 71: 829-38.

103. Wang H, Wang Q, Tian Z, et al. Right ventricular function is associated with quality of life in patients with systemic lupus erythe- matosus associated pulmonary arterial hypertension. Hear Lung Circ. 2019; 28: 165563.

104. Tarazi M, Gaffney RG, Pearson D, et al. Fatigue in systemic lupus erythematosus and other autoimmune skin diseases. Br J Dermatol. 2019; 180: 1468-72.

105. Groot N, Shaikhani D, Teng YKO, et al. Long-term clinical outcomes in a cohort of adults with childhood-onset systemic lupus erythematosus. Arthritis Rheumatol. 2019; 71: 290-301.

106. Xie X, Song Y, Yang H, et al. Effects of transitional care on self-care, readmission rates, and quality of life in adult patients with systemic lupus erythematosus: a randomized controlled trial. Arthritis Res Ther. 2018; 20: 1-8.

107. Parodis I, Lopez Benavides AH, Zickert A, et al. The impact of belimumab and rituximab on health-related quality of life in patients with systemic lupus erythematosus. Arthritis Care Res. 2019; 71: 811-21.

108. Jolly M, Sequeira W, Block JA, et al. Sex differences in quality of life in patients with systemic lupus erythematosus. Arthritis Care Res. 2019; 71: 1647-52.

109. Figueiredo-Braga M, Cornaby C, Bernardes $\mathrm{M}$, et al. Correlation between physical markers and psychiatric health in a Portuguese systemic lupus erythematosus cohort: The role of suffering in chronic autoimmune disease. PLoS One. 2018; 13: 1-16.

110. Waldheim E, Ajeganova S, Bergman S, et al. Variation in pain related to systemic lupus erythematosus (SLE): a 7-year follow-up study. Clin Rheumatol. 2018; 37: 1825-34.

111. Pascual-Ramos V, Contreras-Yáñez I, Valencia-Quiñones KR, Romero-Díaz J. Rheumatoid arthritis patients achieved better quality of life than systemic lupus erythematosus patients at sustained remission. Clin Exp Rheumatol. 2018; 36: 619-26.

112. Merrill JT, Shanahan WR, Scheinberg M, et al. Phase III trial results with blisibimod, a selective inhibitor of B-cell activating factor, in subjects with systemic lupus erythematosus (SLE): Results from a randomised, double-blind, placebo-controlled trial. Ann Rheum Dis. 2018; 77: 883-9.

113. Margiotta DPE, Basta F, Dolcini G, et al. Physical activity and sedentary behavior in patients with systemic lupus erythematosus. PLoS One. 2018; 13: 1-16.

114. Kasturi S, Szymonifka J, Burket JC, et al. Feasibility, validity, and reliability of the 10item patient reported outcomes measurement information system global health short form in outpatients with systemic lupus erythematosus. J Rheumatol. 2018; 45: 397-404.

115. Zhao Q, Chen H, Yan H, et al. The correlations of psychological status, quality of life, 
self-esteem, social support and body image disturbance in Chinese patients with systemic lupus erythematosus. Psychol Heal Med. 2018; 23: 779-87.

116. Nowicka-Sauer K, Hajduk A, KujawskaDanecka $H$, et al. Illness perception is significantly determined by depression and anxiety in systemic lupus erythematosus. Lupus. 2018; 27: 454-60.

117. Aziz MM, Galal MAA, Elzohri MH, et al. Cross-cultural adaptation and validation of systemic lupus erythematosus quality of life questionnaire into Arabic. Lupus. 2018; 27: 780-7.

118. Seguier J, Jouve E, Bobot M, et al. Paradoxical association between blood modular interferon signatures and quality of life in patients with systemic lupus erythematosus. Rheumatology (Oxford). 2020; 59: 1975-83.

119. Margiotta DPE, Fasano S, Basta F, et al. The association between duration of remission, fatigue, depression and health-related quality of life in Italian patients with systemic lupus erythematosus. Lupus. 2019; 28: 1705-11.

120. Jolly M, Annapureddy N, Arnaud L, Devilliers H. Changes in quality of life in relation to disease activity in systemic lupus erythematosus: post-hoc analysis of the BLISS-52 Trial. Lupus. 2019; 28: 1628-39.

121. Pinto B, Jolly M, Dhooria A, et al. Hindi LupusPRO: cross cultural validation of disease specific patient reported outcome measure of lupus. Lupus. 2019; 28: 1534-40.

122. Pinto B, Grover S, Dhooria A, et al. Sexual functioning and its correlates in premenopausal married Indian women with systemic lupus erythematosus. Int J Rheum Dis. 2019; 22: 1814-9.

123. Gholizadeh S, Azizoddin DR, Mills SD, et al. Body image mediates the impact of pain on depressive symptoms in patients with systemic lupus erythematosus. Lupus. 2019; 28: 1148-53.

124. Wu ML, Tsai JC, Yu KH, Chen JJ. Effects of physical activity counselling in women with systemic lupus erythematosus: a randomized controlled trial. Int J Nurs Pract. 2019; 25: $1-11$.

125. Živković V, Mitić II B, Stamenković B, et al. Analysis on the risk factors for organ damage in patients with systemic lupus erythematosus: a cross-sectional single-center experience. Sao Paulo Med J. 2019; 137: 155-61.

126. Kasturi S, Szymonifka J, Berman JR, et al. Responsiveness of the patient-reported outcomes measurement information system global health short form in outpatients with systemic lupus erythematosus. Arthritis Care Res. 2020; 72: 1282-8.

127. Poomsalood N, Narongroeknawin P, Chaiamnuay S, et al. Prolonged clinical re- mission and low disease activity statuses are associated with better quality of life in systemic lupus erythematosus. Lupus. 2019; 28 : 1189-96.

128. Ugarte-Gil MF, Pons-Estel GJ, Vila LM, et al. Time in remission and low disease activity state (LDAS) are associated with a better quality of life in patients with systemic lupus erythematosus: Results from LUMINA (LXXIX), a multiethnic, multicentre US cohort. RMD Open. 2019; 5: 1-4.

129. Ugarte-Gil MF, Gamboa-Cárdenas RV, Reátegui-Sokolova $\mathrm{C}$, et al. Better healthrelated quality of life in systemic lupus erythematosus predicted by low disease activity state/remission: data from the Peruvian Almenara lupus cohort. Arthritis Care Res. 2020; 72: 1159-62.

130. Margiotta DPE, Fasano S, Basta F, et al. Clinical features of patients with systemic lupus erythematosus according to healthrelated quality of life, entity of pain, fatigue and depression: a cluster analysis. Clin Exp Rheumatol. 2019; 37: 535-9.

131. Goswami RP, Chatterjee R, Ghosh P, et al. Quality of life among female patients with systemic lupus erythematosus in remission. Rheumatol Int. 2019; 39: 1351-8.

132. Feng C, He Q, Wu Y, et al. Psychometric properties of fatigue severity scale in Chinese systemic lupus erythematosus patients. Health Qual Life Outcomes. 2019; 17: 15-20.

133. Abu Bakar F, Shaharir SS, Mohd R, et al. Work disability in a multi-ethnic Malaysian systemic lupus erythematosus cohort: a cross-sectional study. Int J Rheum Dis. 2019; 22: 1002-7.

134. Horisberger A, Courvoisier D, Ribi C. The fatigue assessment scale as a simple and reliable tool in systemic lupus erythematosus: a cross-sectional study. Arthritis Res Ther. 2019; 21: 1-8.

135. Román Ivorra JA, Fernández-Llanio-Comella N, San-Martín-Álvarez A, et al. Health-related quality of life in patients with systemic lupus erythematosus: a Spanish study based on patient reports. Clin Rheumatol. 2019; 38: 1857-64.

136. Gavilán-Carrera B, Da Silva JG, Vargas-Hitos JA, et al. Association of physical fitness components and health-related quality of life in women with systemic lupus erythematosus with mild disease activity. PLoS One. 2019; 14: 1-17.

137. Legge A, Kirkland S, Rockwood K, et al. Evaluating the properties of a frailty index and its association with mortality risk among patients with systemic lupus erythematosus. Arthritis Rheumatol. 2019; 71: 1297-307.

138. Park EH, Strand V, Oh YJ, et al. Health-related quality of life in systemic sclerosis compared 
with other rheumatic diseases: a cross-sectional study. Arthritis Res Ther. 2019; 21: 1-10.

139. Conceição CTM, Meinão IM, Bombana JA, Sato EI. Psychoanalytic psychotherapy improves quality of life, depression, anxiety and coping in patients with systemic lupus erythematosus: a controlled randomized clinical trial. Adv Rheumatol (London, England). 2019; 59: 4.

140. Castellano Rioja E, Valero-Moreno S, del Carmen Giménez-Espert M, Prado-Gascó $\mathrm{V}$. The relations of quality of life in patients with lupus erythematosus: regression models versus qualitative comparative analysis. $\mathrm{J}$ Adv Nurs. 2019; 75: 1484-92.

141. Delis PC. Uncertainty and quality of life in systemic lupus erythematosus: a cross-sectional study. Rehabil Nurs. 2019; 44: 2-10.

142. Katz P, Wan GJ, Daly P, et al. Patient-reported flare frequency is associated with diminished quality of life and family role functioning in systemic lupus erythematosus. Qual Life Res. 2020; 29: 3251-61.

143. Ratanasiripong NT, Ratanasiripong P. Predictive factors of quality of life among systemic lupus erythematosus patients in Thailand: a web-based cross-sectional study. Qual Life Res. 2020; 29: 2415-23.

144. Legge A, Kirkland S, Rockwood K, et al. Prediction of damage accrual in systemic lupus erythematosus using the systemic lupus international collaborating clinics frailty index. Arthritis Rheumatol. 2020; 72: 658-66.

145. Revenson TA, Schiaffino KM, Deborah Majerovitz S, Gibofsky A. Social support as a double-edged sword: The relation of positive and problematic support to depression among rheumatoid arthritis patients. Soc Sci Med. 1991; 33: 807-13.

146. Varni JW, Burwinkle TM, Seid M, Skarr D. The PedsQL ${ }^{\mathrm{TM}} * 4.0$ as a pediatric population health measure: Feasibility, reliability, and validity. Ambul Pediatr. 2003; 3: 329-41.

147. Rothrock NE, Hays RD, Spritzer K, et al. Relative to the general US population, chronic diseases are associated with poorer health-related quality of life as measured by the Patient-Reported Outcomes Measurement Information System (PROMIS). J Clin Epidemiol. 2010; 63: 1195-204.

148. Moorthy LN, Peterson MGE, Baratelli M, et al. Multicenter validation of a new quality of life measure in pediatric lupus. Arthritis Care Res. 2007; 57: 1165-73.

149. Meiorin S, Pistorio A, Ravelli A, et al. Validation of the childhood health assessment questionnaire in active juvenile systemic lupus erythematosus. Arthritis Rheum. 2008; 59: 1112-9.

150. de Raaij EJ, Schröder C, Maissan FJ, et al. Cross-cultural adaptation and measurement properties of the brief illness perception questionnaire-dutch language version. Man Ther. 2012; 17: 330-5.

151. Williams EM, Ortiz K, Flournoy-Floyd M, et al. Systemic lupus erythematosus observations of travel burden: A qualitative inquiry. Int J Rheum Dis. 2015; 18: 751-60.

152. Elera-Fitzcarrald C, Vega K, GamboaCárdenas RV, et al. Reliability of visual analog scale and numeric rating scale for the assessment of disease activity in systemic lupus erythematosus. JCR J Clin Rheumatol. 2020; 26: S170-3.

153. Beiza BL, Henke CJ, Yeun E, et al. Correlates of fatigue in older adults with rheumatoid arthritis. Nurs Res. 1993; 42: 93-9.

154. Wang S, Wu B, Zhu L, et al. Construct and criterion validity of the euro Qol-5D in patients with systemic lupus erythematosus. PLoS One. 2014; 9: e98883.

155. Maneeton B, Maneeton N, Louthrenoo W. Cognitive deficit in patients with systemic lupus erythematosus. Asian Pacific J Allergy Immunol. 2010; 28: 77-83.

156. Buysse DJ, Reynolds CF, Monk TH, et al. The Pittsburgh sleep quality index: A new instrument for psychiatric practice and research. Psychiatry Res. 1989; 28: 193-213.

157. Ferrucci L, Koh C, Bandinelli S, Guralnik JM. Measuring disability. Pract Geriatr. 2007: 255-70.

158. Zigmond AS, Snaith RP. The hospital anxiety and depression scale. Acta Psychiatr Scand. 1983; 67: 361-70.

159. Cohen S, Kamarck T, Mermelstein R. A global measure of perceived stress. J Health Soc Behav. 1983; 24: 385-96.

160. Hunt SM, McKenna SP, McEwen J, et al. The Nottingham health profile: subjective health status and medical consultations. Soc Sci Med Part A Med Psychol Med. 1981; 15: 221-9.

161. Slade GD, Spencer AJ. Development and evaluation of the oral health impact profile. Community Dent Health. 1994; 11: 3-11.

162. Rosch PJ. Insomnia, psychological assessment and management. Charles M. Morin, Guilford Press, New York, 1993. No. of pages: 238. Price: \$26.95. Stress Med. 1994; 10 : 69-70.

163. Kroenke K, Spitzer RL, Williams JBW. The PHQ-9: Validity of a brief depression severity measure. J Gen Intern Med. 2001; 16: 606-13.

164. Yazdany J. Health-related quality of life measurement in adult systemic lupus erythematosus: Lupus Quality of Life (LupusQoL), Systemic Lupus Erythematosus-Specific Quality of Life Questionnaire (SLEQOL), and Systemic Lupus Erythematosus Quality of Life Questionnaire (L-QoL). Arthritis Care Res. 2011; 63(SUPPL. 11). 
165. Hassett AL, Li T, Radvanski DC, et al. Assessment of health-related family role functioning in systemic lupus erythematosus: Preliminary validation of a new measure. Arthritis Care Res (Hoboken). 2012; 64: 1341-8.

166. Hennessy CH, Moriarty DG, Zack MM, et al. Measuring health-related quality of life for public health surveillance. Public Health Rep. 1994; 109: 665-72.

167. Spitzer RL, Kroenke K, Williams JBW, Löwe B. A brief measure for assessing generalized anxiety disorder: The GAD-7. Arch Intern Med. 2006; 166: 1092-7.

168. Ferreira LN, Ferreira PL, Pereira LN, et al.
Exploring the consistency of the SF-6D. Value Heal. 2013; 16: 1023-31.

169. Castellano Rioja E. Calidad de vida en pacientes con lupus eritematoso. Nueva escala de valoración. 2015 (cited 2021 Feb 24); Available from: https://dialnet.unirioja.es/servlet/tesis?co digo $=158963 \&$ info $=$ resumen\&idioma=SPA

170. Coyne KS, Thompson CL, Lai JS, Sexton CC. An overactive bladder symptom and health-related quality of life short-form: Validation of the OAB-q SF. Neurourol Urodyn. 2015; 34: 255-63.

171. Chalder T, Berelowitz G, Pawlikowska T, et al. Development of a fatigue scale. J Psychosom Res. 1993; 37: 147-53. 